\title{
A Fuzzy Linear Regression Model With Functional Predictors And Fuzzy Responses
}

\section{Gholamreza Hesamian}

Payame Noor University

Mohammad Ghasem Akbari ( $\nabla$ g_z_akbari@yahoo.com )

Birjand University, Iran

\section{Research Article}

Keywords: Goodness-of-fit measure, functional fuzzy number, SCAD penalty, functional regression model.

Posted Date: April 26th, 2021

DOI: https://doi.org/10.21203/rs.3.rs-207762/v1

License: (c) (1) This work is licensed under a Creative Commons Attribution 4.0 International License. Read Full License 


\title{
A Fuzzy Linear Regression Model With Functional Predictors And Fuzzy Responses
}

\author{
Gholamreza Hesamian and Mohammad Ghasem Akbari \\ Department of Statistics, Payame Noor University, Tehran 19395-3697, Iran, \\ Department of Mathematical Sciences, University of Birjand, Birjand, Iran, \\ email: gh.hesamian@pnu.ac.ir, g_z_akbari@birjand.ac.ir
}

\begin{abstract}
A novel functional regression model was introduced, where the predictor was a curve linked to a scalar fuzzy response variable. An absolute error-based penalized method with SCAD loss function was proposed to evaluate the unknown components of the model. For this purpose, a concept of fuzzy-valued function was developed and discussed. Then, a fuzzy large number notion was proposed to estimate the fuzzyvalued function. Some common goodness-of-fit criteria were also used to examine the performance of the proposed method. Efficiency of the proposed method was then evaluated through two numerical examples, including a simulation study and an applied example in the scope of watershed management. The proposed method was also compared with several common fuzzy regression models in cases where the functional data was converted to scalar ones.
\end{abstract}

Keywords: Goodness-of-fit measure, functional fuzzy number, SCAD penalty, functional regression model.

\section{Introduction}

As the most basic and commonly used statistical technique, multiple regression analysis is utilized to estimate the relationships between one or more predictors (independent variables) and a response (dependent variable). Recently, many techniques have been proposed by different authors to combine the conventional statistical regression models with the concept of fuzzy set theory. In this regard, Chukhrova and Johannssen [1] provided a comprehensive systematic review of thenavailable methodologies and applications focused on fuzzy regression analysis as of 2019. Such studies can be classified as (1) possibilistic approaches, where linear and non-linear programming methods are minimized by minimizing the total 
spread of their fuzzy parameters, subject to the support observations at some specific levels (see for example Refs. $[2,3,4,5,6,7,8,9,10,11,12,13,14]$ ), (2) fuzzy least squares and fuzzy least absolutes parametric/non-parametric methods, where the gap between the predicted fuzzy values and available fuzzy data is minimized with regard to various distance measures between two fuzzy numbers, covering the most commonly used linear and non-linear models (see for instance Refs. $[15,16,17,18,19,20,21,22,23,24,25,26,27,28,29])$, and (3) machine learning techniques, like evolutionary algorithms [30, 31, 32, 33, 34], support vector machines [35, 36, 37, 38], and neural networks embedded in fuzzy regression analysis $[39,40,41,42,43]$, where the ideas and terminology relevant to biological evolution are used, such as mutation, recombination, reproduction and selection. Here the candidate solutions of the optimization problem represent individuals in a population. Accordingly, a fitness function is used to determine the quality of some solutions of the optimization problem with individuals in the underlying population. Cheng and Lee [44] investigated the two most basic non-parametric regression techniques, namely k-nearest neighbor smoothing and kernel smoothing, for a problem with crisp input and fuzzy output. They further formulated an algorithm to select the best smoothing parameters based on minimization of cross-validation criteria. Wang et al. [45] proposed a fuzzy non-parametric model with crisp input and $L R$ fuzzy output based on the local linear smoothing technique with a cross-validation procedure to select the optimal value of the smoothing parameter to fit the model. Additionally, Hesamian and Akbari [47] and Yang and Yin [47] proposed some fuzzy multiple regression model with fuzzy varying coefficients based on exact predictors and fuzzy responses.

All of the above mentioned fuzzy regression models relied on non-functional data. However, functional regression analysis [48] has received considerable attentions in various fields of application $[49,50,51]$. The basic idea behind functional regression analysis is to express each predictor in a repeatedly measured set of data as a smooth function and then draw information from the collection of the functional data. The term "functional" data traditionally refers to the data measured over an interval or a higher dimensional domain. Such data is recorded at discrete times to form a continuous function in order to (1) allow record evaluation at any point in time, (2) evaluate rates of change, (3) reduce noise, and (4) allow registration onto a common time-scale. From another pont of view, regression models with functional data can be classified into three classes: those with (1) functional predictor(s) and scalar response [52, 53], (2) scalar predictor(s) and functional response [54, 55, 48, 49, 56, $57,58,59,60,61]$ and (3) functional predictor(s) and functional response [62, 63, 64]. Many of these methods are direct extensions of the classical least squares, principal 
component, and partial least-squares procedures.

Previous studies on fuzzy regression analysis have been conducted on the basis of non-functional scalar/fuzzy quantities with exact/fuzzy or exact/fuzzy-valued (varying) coefficients. In this paper, however, a fuzzy functional linear regression modeling strategy is proposed based on functional predictors and a $L R$-fuzzy response and fuzzy varying coefficients. For many experts, a simple way to capture imprecision in a vague process is to express that as an $L R$-fuzzy number. Therefore, the $L R$-fuzzy numbers play an important role in many real-life applications of fuzzy inferences. To evaluate the unknown components of the proposed fuzzy functional coefficients, a criteria selection model is herein proposed via absolute error regularization and SCAD penalty.

The rest of this paper is organized as follows: Section 2 reviews some general concepts relevant to the fuzzy numbers. In Section 3, a methodology is proposed to estimate the fuzzy coefficients of a fuzzy regression model with functional predictors and fuzzy responses. A hybrid algorithm is also represented to evaluate the components of the proposed fuzzy functional regression model. Section 4 presents three numerical examples to evaluate the performance of the proposed method compared to other fuzzy multiple/non-linear/non-parametric regression methods in terms of some common performance measures. Finally, the main contributions of this paper are summarized in Section 5.

\section{Fuzzy numbers}

This section reviews some basic definitions of fuzzy numbers based on $[65,66]$. A fuzzy set $\widetilde{A}$ of $\mathbb{R}$ (the real line) is defined by its membership function $\mu_{\widetilde{A}}: \mathbb{R} \rightarrow$ $[0,1]$. In addition, a fuzzy set $\widetilde{A}$ of $\mathbb{R}$ is called a fuzzy number $(\mathbf{F N})$ if it is normal, i.e. there is a unique $x_{\widetilde{A}}^{*} \in \mathbb{R}$ so that $\mu_{\widetilde{A}}\left(x_{\widetilde{A}}^{*}\right)=1$; and for every $\alpha \in[0,1]$, the set $\widetilde{A}[\alpha]=\left\{x \in \mathbb{R}: \mu_{\widetilde{A}}(x) \geq \alpha\right\}$ is a nonempty compact interval in $\mathbb{R}$. This interval is denoted by $\widetilde{A}[\alpha]=\left[\widetilde{A}_{\alpha}^{L}, \widetilde{A}_{\alpha}^{U}\right]$, where $\widetilde{A}_{\alpha}^{L}=\inf \{x: x \in \widetilde{A}[\alpha]\}$ and $\widetilde{A}_{\alpha}^{U}=\sup \{x: x \in$ $\widetilde{A}[\alpha]\}$. The set of all fuzzy numbers is denoted by $\mathbb{F}(\mathbb{R})$. It is worth noting that fuzzy numbers are approximate assessments, given by experts and accepted by decisionmakers when access to more accurate values is either impossible or unnecessary. To simplify the fuzzy numbers representation and handling, several authors have captured the information contained in a (unimodal) fuzzy number using a functional parametric form known as $L R$-fuzzy number $\widetilde{A}=\left(a ; l_{a}, r_{a}\right)_{L R}$. The membership 
function of a $L R$-fuzzy number $\widetilde{A}$ is defined by:

$$
\mu_{\widetilde{A}}(x)= \begin{cases}L\left(\frac{a-x}{l_{a}}\right), & x \leq a, \\ R\left(\frac{x-a}{r_{a}}\right), & x>a,\end{cases}
$$

where $a \in \mathbb{R}, l_{a}>0$ and $r_{a}>0$ are called the mean value, left and right spreads of $\widetilde{A}$, respectively. The shape function $L$ (or $R$ ) is a decreasing function from $\mathbb{R}^{+} \rightarrow[0,1]$ such that

1. $L(0)=1$,

2. $L(x)<1$ for every $x>0$,

3. $L(x)>0$ for every $x<1$,

4. $L(1)=0($ or $L(x)>0$ for any $x \in \mathbb{R}$ and $L(+\infty)=0)$.

An $L R$-number has been applied in various problems as a general model function of imprecision. In this paper, we employed the most commonly used $L R$-fuzzy numbers (with $L(x)=R(x)=\max \{0,1-x\}$ ) so-called triangular fuzzy numbers (TFNs), to handle the imprecision in data set during numerical evaluations. The membership function of a triangular fuzzy number, denoted by $\widetilde{A}=\left(a ; l_{a}, r_{a}\right)_{T}$, is given by:

$$
\mu_{\widetilde{A}}(x)=\left\{\begin{array}{lr}
\frac{x-\left(a-l_{a}\right)}{l_{a}}, & a-l_{a} \leq x \leq a, \\
\frac{a+r_{a}-x}{r_{a}}, & a<x \leq a+r_{a}, \\
0, & x \in \mathbb{R}-\left(a-l_{a}, a+r_{a}\right) .
\end{array}\right.
$$

Definition 2.1. [65] For a given $\widetilde{A} \in \mathbb{F}(\mathbb{R})$, the mapping $\widetilde{A}_{\alpha}:[0,1] \rightarrow \mathbb{R}$ is called $\alpha$-values of $\widetilde{A}$ defined as follows:

$$
\widetilde{A}_{\alpha}= \begin{cases}\widetilde{A}^{L}[2 \alpha] & \alpha \in[0,0.5], \\ \widetilde{A}^{U}[2(1-\alpha)] & \alpha \in(0.5,1],\end{cases}
$$

where $\widetilde{A}^{L}[\alpha]$ and $\widetilde{A}^{U}[\alpha]$ denote the lower and upper limits of $\alpha$-cuts of $\widetilde{A}$.

Example 2.1. Let $\widetilde{A}=\left(a ; l_{a}, r_{a}\right)_{L R}$ be an LR-fuzzy number. From Definition 2.1, one finds

$$
\widetilde{A}_{\alpha}= \begin{cases}a-l_{a} L^{-1}(2 \alpha), & 0.0 \leq \alpha \leq 0.5 \\ a+r_{a} R^{-1}(2(1-\alpha)), & 0.5 \leq \alpha \leq 1.0\end{cases}
$$

For instance, 
1. If $\widetilde{A}=\left(a ; l_{a}, r_{a}\right)_{T}$ is a triangular fuzzy number, then:

$$
\widetilde{A}_{\alpha}= \begin{cases}\left(a-l_{a}\right)+2 l_{a} \alpha, & 0.0 \leq \alpha \leq 0.50 \\ a+r_{a}-2 r_{a}(1-\alpha), & 0.50<\alpha \leq 1.0 .\end{cases}
$$

2. Let $\widetilde{A}=(\mu, \sigma)_{G}$ be a Gaussian $\boldsymbol{F} \boldsymbol{N}$ with the membership function of $\mu_{\widetilde{A}}(x)=$ $\exp \left(-0.5[(x-\mu) / \sigma]^{2}\right)$, then:

$$
\widetilde{A}_{\alpha}= \begin{cases}\mu-\sigma \sqrt{-2 \ln (2 \alpha)}, & 0.0<\alpha \leq 0.50, \\ \mu+\sigma \sqrt{-2 \ln (2(1-\alpha))}, & 0.50<\alpha<1.0 .\end{cases}
$$

Remark 2.1. Since $\widetilde{A}_{\alpha}$ is a decreasing function of $\alpha$, the relationship between $\alpha$ values and $\alpha$-cuts can be expressed as:

$$
\widetilde{A}[\alpha]=\left[\widetilde{A}^{L}[\alpha], \widetilde{A}^{U}[\alpha]\right]=\left[\widetilde{A}_{\alpha / 2}, \widetilde{A}_{1-\alpha / 2}\right] .
$$

Therefore, having a sequence of $\alpha$-values $\left\{\widetilde{A}_{\alpha}\right\}_{\alpha \in[0,1]}$, the membership function of $\widetilde{A}$ can be evaluated as follows:

$$
\mu_{\widetilde{A}}(x)=\sup \left\{\alpha \in[0,1]: x \in\left[\widetilde{A}_{\alpha / 2}, \widetilde{A}_{1-\alpha / 2}\right]\right\}, x \in \mathbb{R} .
$$

In addition, for all $\widetilde{A}, \widetilde{B} \in \mathbb{F}(\mathbb{R}), \lambda \in \mathbb{R}$ and $\alpha \in[0,1]$, the addition and scalar multiplication operations between $\widetilde{A}$ and $\widetilde{B}$ can be evaluated as follows:

$$
\begin{aligned}
& (\widetilde{A} \oplus \widetilde{B})_{\alpha}=\widetilde{A}_{\alpha}+\widetilde{B}_{\alpha}, \\
& (\lambda \otimes \widetilde{A})_{\alpha}=\left\{\begin{array}{lll}
\lambda \widetilde{A}_{\alpha} & \text { if } \lambda>0, \\
\lambda \widetilde{A}_{1-\alpha} & \text { if } \quad \lambda<0 .
\end{array}\right.
\end{aligned}
$$

Such arithmetic operations will be applied to suggest a fuzzy multivariate regression model in next section.

Definition 2.2. An $L_{p}$ distance measure between two fuzzy numbers $\boldsymbol{F} \boldsymbol{N} s \widetilde{A}$ and $\widetilde{B}$ is defined as

$$
d_{p}(\widetilde{A}, \widetilde{B})= \begin{cases}\left(\int_{0}^{1} g(\alpha)\left|\widetilde{A}_{\alpha}-\widetilde{B}_{\alpha}\right|^{p} d \alpha\right)^{1 / p}, & p \geq 1 \\ \sup _{\alpha \in[0,1]}\left|\widetilde{A}_{\alpha}-\widetilde{B}_{\alpha}\right|, & p=\infty\end{cases}
$$

where

$$
g(\alpha)= \begin{cases}4 \alpha & 0 \leq \alpha \leq 0.5 \\ 4(1-\alpha) & 0.5 \leq \alpha \leq 1\end{cases}
$$


Any three FNs $\widetilde{A}, \widetilde{B}$ and $\widetilde{C}$ satisfy the following conditions:

- $d_{p}(\widetilde{A}, \widetilde{B})=0$ if and only if $\widetilde{A}=\widetilde{B}$

- $d_{p}(\widetilde{A}, \widetilde{B})=d_{p}(\widetilde{B}, \widetilde{A})$

- $d_{p}(\widetilde{A}, \widetilde{C}) \leq\left(d_{p}(\widetilde{A}, \widetilde{B})+d_{p}(\widetilde{B}, \widetilde{C})\right)$.

It should be noted that $g(\alpha)$ also modifies the square error distance between the two FNs $\widetilde{A}$ and $\widetilde{B}$ since it focuses on the values near the centers rather than tails. This distance measure is used to evaluate the unknown components of the proposed fuzzy functional regression model and performances of the proposed fuzzy regression model compared to other fuzzy regression models.

Definition 2.3. $\widetilde{A}(t)$ is said to be a (continuous) fuzzy-valued function on $[a, b]$ if $\widetilde{A}(t)$ is a $\boldsymbol{F N}$ for any $t \in[a, b]$.

Definition 2.4. Let $\widetilde{A}(t)$ be a fuzzy-functional-valued on $[a, b]$. The integration of $\widetilde{A}($.$) over [a, b]$ is defined to be a fuzzy number $\widetilde{\mathfrak{A}}$ with the following $\alpha$-values:

$$
(\widetilde{\mathfrak{A}})_{\alpha}=\int_{a}^{b}(\widetilde{A}(t))_{\alpha} d t .
$$

Example 2.2. If $\widetilde{A}(t)=(0.5 \sin (\pi t) ; 0.1 t)_{L R}$ with $L(x)=\exp (-x / 2)$ and $R(x)=$ $\max \{0,1-x\}$ and $\widetilde{B}(t)=(t ; 1 /(1+t))_{G}$ then, the plots of $\widetilde{\mathfrak{A}}$ and $\widetilde{\mathfrak{B}}$ on $[0,1]$ are shown in Fig. 1. In addition,

$$
\begin{gathered}
d_{2}(\widetilde{\mathfrak{A}}, \widetilde{\mathfrak{B}})=\left(\int_{0}^{1} g(\alpha)\left|\widetilde{\mathfrak{A}}_{\alpha}-\widetilde{\mathfrak{B}}_{\alpha}\right|^{2} d \alpha\right)^{1 / 2}=0.6342, \text { and } \\
d_{1}(\widetilde{\mathfrak{A}}, \widetilde{\mathfrak{B}})=\int_{0}^{1} g(\alpha)\left|\widetilde{\mathfrak{A}}_{\alpha}-\widetilde{\mathfrak{B}}_{\alpha}\right| d \alpha=0.6195
\end{gathered}
$$

Lemma 2.1. Let $c, d \in \mathbb{R}$ and $\widetilde{A}($.$) and \widetilde{B}($.$) be two fuzzy-valued functions. Then,$

$$
\int((c \otimes \widetilde{A}(t)) \oplus(d \otimes \widetilde{B}(t))) d t=(c \otimes \widetilde{\mathfrak{A}}) \oplus(d \otimes \widetilde{\mathfrak{B}}),
$$

where $\widetilde{\mathfrak{A}}=\int_{0}^{1}\left(\widetilde{A}(t) d t\right.$ and $\widetilde{\mathfrak{B}}=\int_{0}^{1}(\widetilde{B}(t) d t$. 

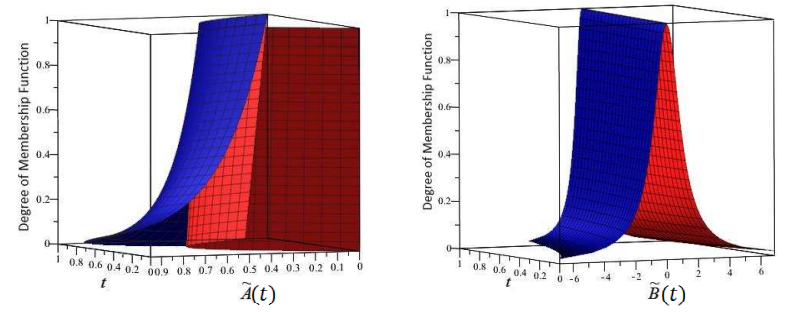

Figure 1: 3D-plot of $\widetilde{A}(t)$ and $\widetilde{B}(t)$ in Example 2.2.

Proof 2.1. According to Definition 2.4 and arithmetic operations of $\alpha$-values fuzzy numbers, it is easy to see that

$$
\begin{gathered}
\left(\int((c \otimes \widetilde{A}(t)) \oplus(d \otimes \widetilde{B}(t))) d t\right)_{\alpha}= \\
\int\left((c \otimes \widetilde{A}(t)) \oplus(d \otimes \widetilde{B}(t))_{\alpha}\right) d t \\
=\int(c \otimes \widetilde{A}(t))_{\alpha} d t+\int(d \otimes \widetilde{B}(t))_{\alpha} d t .
\end{gathered}
$$

Now, note that

$$
\int(c \otimes \widetilde{A}(t))_{\alpha} d t= \begin{cases}c \int \widetilde{A}(t)_{\alpha} d t & c \geq 0, \\ c \int \widetilde{A}(t)_{1-\alpha} d t & c<0,\end{cases}
$$

and

$$
\int(d \otimes \widetilde{B}(t))_{\alpha} d t= \begin{cases}d \int \widetilde{B}(t)_{\alpha} d t & d \geq 0, \\ d \int \widetilde{B}(t)_{1-\alpha} d t & d<0 .\end{cases}
$$

Therefore, $\int(c \otimes \widetilde{A}(t)) d t=c \otimes \widetilde{\mathfrak{A}}$ and $\int(d \otimes \widetilde{B}(t)) d t=d \otimes \widetilde{\mathfrak{B}}$ which completes the proof.

Here a notion of large numbers is developed for a fuzzy-valued function of $\widetilde{A}(t)$.

Lemma 2.2. Let $\widetilde{A}(t)$ be a fuzzy-valued function on $[0,1]$. If $U_{1}, U_{2}, \ldots, U_{N}$ are independent random variables uniformly distributed over the interval $[a, b]$, then

$$
\lim _{N \rightarrow \infty} P\left(d_{\infty}\left(\int_{a}^{b} \widetilde{A}(t) d t,\left(\frac{b-a}{N}\right) \otimes \oplus_{i=1}^{N} \widetilde{A}\left(U_{i}\right)\right)=0\right)=1
$$


Proof 2.2. According to Definition 2.2, first note that:

$$
\begin{gathered}
d_{\infty}\left(\int_{a}^{b} \widetilde{A}(t) d t,\left(\frac{b-a}{N}\right) \otimes \bigoplus_{i=1}^{N} \widetilde{A}\left(U_{i}\right)\right)= \\
\sup _{\alpha \in[0,1]}\left|\int_{a}^{b}(\widetilde{A}(t))_{\alpha} d t-\left(\frac{b-a}{N}\right) \sum_{i=1}^{N}\left(\widetilde{A}\left(U_{i}\right)\right)_{\alpha}\right| .
\end{gathered}
$$

By strong law of large numbers [67], we know that

$$
\lim _{N \rightarrow \infty} P\left(\left|\int_{a}^{b}(\widetilde{A}(t))_{\alpha} d t-\left(\frac{b-a}{N}\right) \sum_{i=1}^{N}\left(\widetilde{A}\left(U_{i}\right)\right)_{\alpha}\right|=0\right)=1,
$$

for any $\alpha \in[0,1]$. This simply concludes that $\lim _{N \rightarrow \infty} P\left(\sup _{\alpha \in[0,1]} \mid \int_{a}^{b}(\widetilde{A}(t))_{\alpha} d t-\right.$ $\left.\left(\frac{b-a}{N}\right) \sum_{i=1}^{N}\left(\widetilde{A}\left(U_{i}\right)\right)_{\alpha} \mid=0\right)=1$. This completes the proof.

Remark 2.2. According to Lemma 2.2, the integration of $\widetilde{A}($.$) over [a, b]$ can be approximated by a Mont Carlo simulation as $\left(\frac{b-a}{N}\right) \otimes \bigoplus_{i=1}^{N} \widetilde{A}\left(U_{i}\right)$, that is:

$$
\int_{a}^{b} \widetilde{A}(t) d t \simeq\left(\frac{b-a}{N}\right) \otimes \bigoplus_{i=1}^{N} \widetilde{A}\left(U_{i}\right)
$$

for a large value of $N$.

\section{Fuzzy functional linear regression model}

Functional data analysis is a fast evolving branch of applied statistics, with the functional regression becoming popular in recent years. In this section, a functional linear regression model with fuzzy functional predictors, fuzzy responses and fuzzy functional coefficients was developed. Denoting the observed data on $\mathrm{n}$ statistical units by $\left(\widetilde{y}_{i}, \boldsymbol{x}_{i}()=.\left(x_{i 1}(.), x_{i 2}(.), \ldots, x_{i p}(.)\right)^{\top}\right)$, consider the following fuzzy functional linear regression model:

$$
\widetilde{y}_{i}=\widetilde{\alpha} \oplus \bigoplus_{j=1}^{d} \int_{a}^{b}\left(\widetilde{\beta}_{j}(t) \otimes x_{i j}(t)\right) d t \oplus \widetilde{\epsilon}_{i}, i=1,2, \ldots, n,
$$

where 
1. $\widetilde{y}_{i}=\left(y_{i} ; l_{y_{i}}, r_{y_{i}}\right)_{L_{i} R_{i}}$ represent fuzzy responses,

2. $\widetilde{\alpha}$ denotes the unknown fuzzy intercept,

3. $\widetilde{\beta}_{j}(t)=\left(\beta_{j}(t) ; l_{\beta_{j}(t)}, r_{\beta_{j}(t)}\right)_{L_{j} R_{j}}$ are the coefficients of the true fuzzy-valued function, and

4. $\widetilde{\epsilon}_{i}$ indicates a fuzzy error term.

Based on the fuzzy law of large numbers in fuzzy domain (Lemma 2.1), the fuzzy functional linear regression model (5) can be converted to a conventional fuzzy linear regression model:

$$
\widetilde{y}_{i}=\widetilde{\alpha} \oplus\left(\frac{(b-a)}{N}\right) \bigoplus_{k=1}^{N} \bigoplus_{j=1}^{d}\left(\widetilde{\beta}_{j}\left(U_{k}\right) \otimes x_{i j}\left(U_{k}\right)\right) \oplus \widetilde{\epsilon}_{i}, i=1,2, \ldots, n,
$$

where $U_{1}, U_{2}, \ldots, U_{N}$ are independent random variables uniformly distributed over the interval $[a, b]$ and $N \in \mathbb{N}$ is a large number.

Remark 3.1. Note that the unknown fuzzy varying coefficients of model (6) can be presented as $\boldsymbol{B}=\left(\boldsymbol{L}_{B}, B, \boldsymbol{R}_{B}\right)^{\top}$, where:

$$
\begin{gathered}
B=\left(\begin{array}{cccc}
\beta_{11} & \beta_{21} & \ldots & \beta_{d 1} \\
\vdots & \vdots & \ldots & \vdots \\
\beta_{N 1} & \beta_{N 2} & \ldots & \beta_{N d}
\end{array}\right) \\
\boldsymbol{L}_{B}=\left(\begin{array}{cccc}
l_{\beta_{11}} & l_{\beta_{12}} & \ldots & l_{\beta_{1 d}} \\
\vdots & \vdots & \ldots & \vdots \\
l_{\beta_{N 1}} & l_{2 \beta_{N 2}} & \ldots & l_{\beta_{N d}}
\end{array}\right), \text { and } \\
\boldsymbol{R}_{B}=\left(\begin{array}{cccc}
r_{\beta_{11}} & r_{\beta_{12}} & \ldots & r_{\beta_{1 d}} \\
\vdots & \vdots & \ldots & \vdots \\
r_{\beta_{N 1}} & r_{2 \beta_{N 2}} & \ldots & r_{\beta_{N d}}
\end{array}\right)
\end{gathered}
$$

where $\beta_{k j}=\beta_{j}\left(U_{k}\right), l_{\beta_{k j}}=l_{\beta_{j}\left(U_{k}\right)}$ and $r_{\beta_{k j}}=r_{\beta_{j}\left(U_{k}\right)}$. 


\subsection{Estimation of unknown fuzzy coefficients and tuning constant}

In order to estimate the fuzzy coefficients of the proposed fuzzy functional regression model (6), a regularization criterion that was originally presnted based on SCAD penalty was extended for the reduced fuzzy multivariate regression model (6), as follows:

$$
(\widetilde{\widehat{\alpha}}, \widehat{\boldsymbol{B}})=\arg \min _{(\widetilde{\alpha}, \boldsymbol{B})}\left\{\sum_{i=1}^{n} d_{1}\left(\widetilde{y}_{i}, \widetilde{y}_{i}^{*}\right)+\sum_{j=1}^{d} \sum_{k=1}^{N} \rho_{\lambda}\left(\left|M_{\widetilde{\beta}_{j}\left(U_{k}\right)}\right|\right)\right\},
$$

where $M_{\widetilde{\beta}_{j k}}=\max \left\{\beta_{j k}, l_{\beta_{j k}}, r_{\beta_{j k}}\right\}$,

$$
\widetilde{y}_{i}^{*}=((b-a) / N) \bigoplus_{k=1}^{N} \bigoplus_{j=1}^{d}\left(\widetilde{\beta}_{j}\left(U_{k}\right) \otimes x_{i j}\left(U_{k}\right)\right),
$$

and

$$
\rho_{\lambda}(|\theta|)=\left\{\begin{array}{lr}
\lambda|\theta| & |\theta|<\lambda, \\
-\left(\theta^{2}-2 \lambda \theta|\theta|+\lambda^{2}\right)^{2} / 5.4 & \lambda<|\theta|<3.7 \lambda, \\
2.35 \lambda^{2} & |\theta|>3.7 \lambda .
\end{array}\right.
$$

According to the proposed fuzzy regression model, the unknown regression coefficients $(\widetilde{\alpha}, \boldsymbol{B})_{\lambda}$ and constant tuning parameter $\lambda$ should be simultaneously estimated based on a set of observed values $\left(\widetilde{y}_{1}, \mathbf{x}_{1}^{\top}().\right), \ldots,\left(\widetilde{y}_{n}, \mathbf{x}_{n}^{\top}().\right)$. Since $(\widetilde{\alpha}, \boldsymbol{B})_{\lambda}$ and $\lambda$ were relaited on one another, beside the optimization problem given in Eq. (10)), the constant tuning parameter can be also evaluated by minimizing the cross validation criterion [68], i.e. $\left(\lambda_{(\widetilde{\alpha}, \boldsymbol{B})}\right)_{\text {opt }}=\arg \min _{\lambda>0} C V(\lambda)$ where:

$$
C V(\lambda)=\frac{1}{n} \sum_{j=1}^{n} d_{2}^{2}\left(\tilde{y}_{j}, \widetilde{\hat{y}}_{\left(\widetilde{\hat{\alpha}}, \widehat{\boldsymbol{B}}_{\lambda}\right)^{(j)}}\right)
$$

in which

$$
\begin{gathered}
\left((\widetilde{\widehat{\alpha}}, \widehat{\boldsymbol{B}})_{\lambda}\right)^{(j)}= \\
\arg \min _{(\widetilde{\alpha}, \boldsymbol{B})}\left\{\sum_{i(\neq j)=1}^{n} d_{1}\left(\widetilde{y}_{i}, \widetilde{y}_{i}^{*}\right)+\sum_{j=1}^{d} \sum_{k=1}^{N} \rho_{\lambda}\left(\left|M_{\widetilde{\beta}_{j}\left(U_{k}\right)}\right|\right)\right\},
\end{gathered}
$$

For this purpose, the values $(\widetilde{\widehat{\alpha}}, \widehat{\boldsymbol{B}})_{\lambda}$ was computed for many values of $\lambda$, looking for an optimal $\lambda_{\text {opt }}$ value that minimizes the one-out cross validation error $(C V)$. Once found, the optimal value of $(\widetilde{\alpha}, \boldsymbol{B})$ was presented by $\left(\widetilde{\widehat{\alpha}}_{o p t}, \widehat{\boldsymbol{B}}_{\text {opt }}\right)$. To this end, the Mathematica software [69] was employed. 
Remark 3.2. To conduct a comparative study with other fuzzy regression models, four widely-used performance criteria for evaluation of fuzzy regression models were used [15]. These included:

1. Root mean square error (RMSE):

$$
R M S E=\sqrt{\frac{\sum_{i=1}^{n} d_{2}^{2}\left(\widetilde{y}_{i}, \widetilde{\widehat{y}}_{i}\right)}{n}} .
$$

2. Mean absolute relative error (MARE):

$$
M A R E=\frac{1}{n} \sum_{i=1}^{n} \frac{\int_{0}^{1}\left|\widetilde{y}_{i}(x)-\widetilde{\widehat{y}}_{i}(x)\right| d x}{\int_{0}^{1} \widetilde{y}_{i}(x) d x} .
$$

3. Mean similarity measure (MSM):

$$
M S M=\frac{1}{n} \sum_{j=1}^{n} S_{U I}\left(\widetilde{\widehat{y}}_{j}, \tilde{y}_{j}\right),
$$

where

$$
S_{U I}\left(\widetilde{\widehat{y}}_{j}, \tilde{y}_{j}\right)=\frac{\operatorname{Card}\left(\widetilde{\widehat{y}}_{j} \cap \tilde{y}_{j}\right)}{\operatorname{Card}\left(\widetilde{\tilde{y}}_{j} \cup \widehat{y}_{j}\right)}
$$

4. Area Under the Receiver Operating Characteristic Curve (AUROCC):

$$
\begin{gathered}
\operatorname{AUROC}_{m}= \\
\frac{1}{2} \sum_{i=m}^{n}\left(R^{n} M S E_{i}+\text { RMSE }_{i-1}\right)\left(\text { coverage }_{i}-\text { coverage }_{i-1}\right),
\end{gathered}
$$

where

$$
R M S E_{i}=\frac{\sum_{j=1}^{i} d_{2}^{2}\left(\widetilde{y}_{j}, \widetilde{y}_{j}\right)}{n}, \text { coverage }_{i}=\frac{i}{n}
$$

In addition, to examine the relationship between $\widetilde{y}$ and $\widetilde{\hat{y}}$ based on their scatter plots, the fuzzy response $(\widetilde{y})$ and the corresponding estimated value $\widetilde{\widehat{y}})$ were converted to defuzzified to $M_{\widetilde{y}}$ and $M_{\widetilde{\hat{y}}}$, respectively, according to the sugeno criteria [70]:

$$
M_{\widetilde{y}}=\frac{\int x \mu_{\widetilde{y}}(x) d x}{\int \mu_{\widetilde{y}}(x) d x}, M_{\widetilde{\widehat{y}}}=\frac{\int x \mu_{\widetilde{\widehat{y}}}(x) d x}{\int \mu_{\widetilde{\widehat{y}}}(x) d x} .
$$


Remark 3.3. It should be pointed out that the classical functional regression methods rely on some regularization basis functions such as B-spline. However, applying such methods in fuzzy domain, need more parameters in the proposed estimation procedure. However, by introducing the concept of fuzzy large number, such procedure was reduced to minimum parameters as much as possible.

\section{Application examples}

The feasibility and effectiveness of the proposed fuzzy functional regression model were examined based on the performance measures explained in Remark 3.2.

Example 4.1. (A simulation study) A set of $m=10$ simulated data set with size of $n=300$ were generated according to the following fuzzy functional regression model:

$$
\widetilde{y}_{i}=\widetilde{\alpha} \oplus \bigoplus_{j=1}^{3} \int_{0}^{1}\left(\widetilde{\beta}_{j}(t) \otimes x_{i j}(t)\right) d t \oplus \widetilde{\epsilon}_{i}, i=1,2, \ldots, n
$$

where

1. $\widetilde{\alpha}=(1 ; 0.2,0.3)_{T}$.

2. $\widetilde{\beta}_{1}(t)=(\sin (\pi t) ; 0.1 t, 0.2 t)_{T}, \widetilde{\beta}_{2}(t)=(\exp (-2 t) ; 0.2 t, 0.4 t)_{L_{1} R_{1}}$, and $\widetilde{\beta}_{3}(t)=$ $\left(-1+2 t-3 t^{2} ; 0.3 t, 0.6 t\right)_{L_{2}}$ where $L_{1}(x)=\exp \left(-x^{2} / 2\right), R_{1}(x)=1 /\left(1+x^{2}\right)$ and $L_{2}(x)=\sqrt{1-x^{2}}$.

3. $x_{i 1}(t)=t(1-t)^{1.5} z_{i 1}+w_{i 1}$ where $z_{i 1} \sim N(0,0.1)$ and $w_{i 1} \sim N(0,0.9)$,

4. $x_{i 2}(t)=10 t(t-0.6)^{2} z_{i 2}+w_{i 2}$ where $z_{i 2} \sim N(0,0.2)$ and $w_{i 2} \sim N(0,0.8)$,

5. $x_{i 3}(t)=\exp (-t) \cos (4 \pi t+0.5) z_{i 3}+w_{i 3}$ where $z_{i 3} \sim N(0,0.3)$ and $w_{i 3} \sim$ $N(0,0.7)$,

6. $\tilde{\epsilon}_{i}=\left(\epsilon_{i} ;\left|\epsilon_{i}\right| /\left(1+\left|\epsilon_{i}\right|\right)\right)_{T}$ where $\epsilon_{i} \sim N(0,1)$.

3D-plots of $\widetilde{\beta}_{1}(t)-\widetilde{\beta}_{3}(t)$ are drawn in Fig. 2. The mean values of the performance measures of the proposed method are reported in Table 1. In particular, consider the performance of the proposed fuzzy functional regression model for the $5^{\text {th }}$ simulated data set (as shown in Table 2 and Fig. 3). The results indicate that $\widetilde{\widehat{\alpha}}=(0.91 ; 0.15,0.36)_{T}$, with the performance of the proposed method also examined by comparing the defuzzified values of $M_{\widetilde{\widehat{y}}}$ and $M_{\widetilde{y}}$. 
Table 1: Mean values of performance measures corresponding to the proposed method and some fuzzy multiple regression techniques for Example 4.1.

\begin{tabular}{|cccccc|}
\hline Performance & $\overline{M S M}$ & $\overline{M A R E}$ & $\overline{R M S E}$ & $\overline{A U C R}_{150}$ & $\overline{A U C R}_{180}$ \\
\hline Result & 0.810 & 14.738 & 10.820 & 31.527 & 23.785 \\
\hline
\end{tabular}

Table 2: Performance measures corresponding to the $5^{\text {th }}$ simulated data set in Example 4.1.

\begin{tabular}{|cccccc|}
\hline Performance & $M S M$ & $M A R E$ & $R M S E$ & $A U C R_{150}$ & $A U C R_{180}$ \\
\hline Result & 0.825 & 12.829 & 9.028 & 25.728 & 19.882 \\
\hline
\end{tabular}

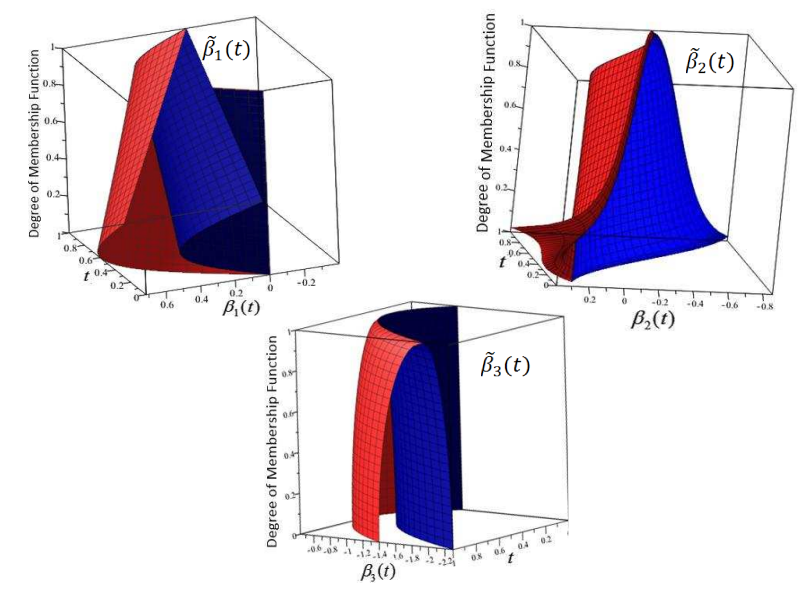

Figure 2: 3D-plot of $\widetilde{\beta}_{1}(t)-\widetilde{\beta}_{3}(t)$ in Example 4.1.

Example 4.2. Prediction of suspended sediment load in a catchments area is very important as it can be used to evaluate the extent of the damage occurred in the catchment, the erosion hazard, and water management. In this example, prediction of the (annual) suspended sediment discharge (ton) based on stream water discharge $\left(m^{3}\right.$ per day) of the Beheshtabad River (Chaharmahal and Bakhtiari Province, Iran) using the proposed fuzzy functional regression model is expected. Cutting through Beheshtabad Village, the river covers an area of $3866 \mathrm{~m}^{2}$ (located between $31^{\circ} 28^{\prime} \mathrm{N}$ and $32^{\circ} 56^{\prime} N$ latitude and $50^{\circ} 36^{\prime} E$ and $51^{\circ} 45^{\prime} E$ longitudes). This is an important stream as it supplies water for agricultural activities, fish farms, hydroelectric power plants, and drinking uses, making it important to monitor the suspended sediment load of this river. We hereby assume that the suspended sediment load is an imprecise quantity that can be expressed as symmetric $\mathbf{T F N}$. The values of suspended 

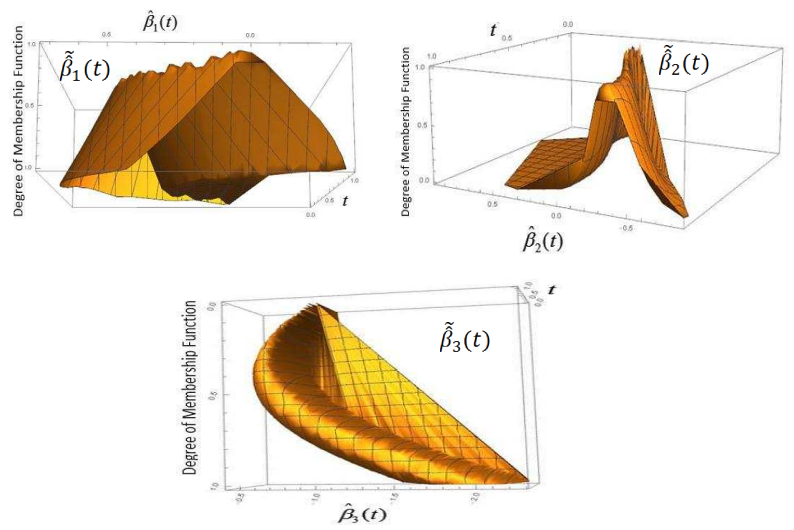

Figure 3: 3D-plot of $\widetilde{\widehat{\beta}}_{1}(t)-\widetilde{\widehat{\beta}}_{3}(t)$ in Example 4.1 .

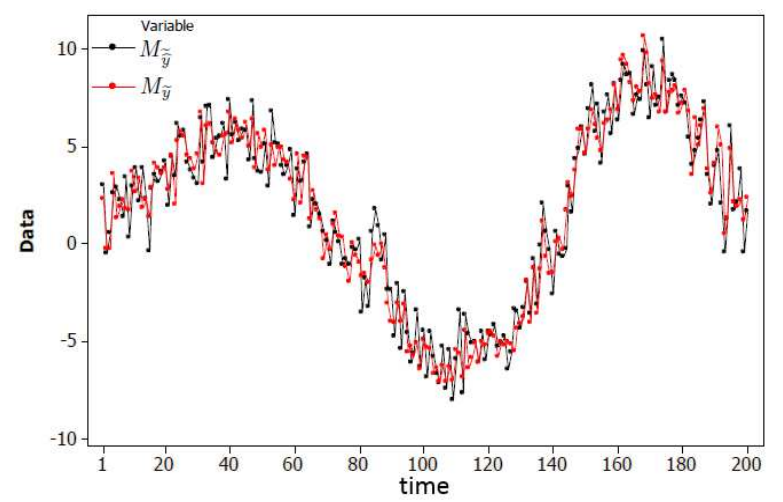

Figure 4: Comparison of $M_{\widetilde{\widehat{y}}}$ versus $M_{\widetilde{y}}$ in Example 4.1 .

sediment load $\left(\widetilde{y}_{i}=\left(y_{i} ; l_{y_{i}}\right)_{T}\right)$ and stream water discharge $x_{i}(t), t=1,2, \ldots, 93$ (in summer, 93 days, as per Solar Hijri calendar) were collected during 2000-2019. The time series plots of $x_{i}(t)$ were smoothed via non-parametric kernel fitting [7r] by Minitab software. For each year, a nonlinear regression model was considered: $x_{j}^{i}=x_{i}(j)+\epsilon_{j}, j=1,2, \ldots, 93$, and $i=2000,2001, \ldots, 2019$. Using the (local) Nadariya-Watson estimator, $x_{i}($.$) can be estimated as \widehat{x}_{i}\left(j^{*}\right)=\sum_{j=1}^{93} w_{h}(j) x_{j}^{i}$ where $w_{h}(j)=K\left(\frac{j-j^{*}}{h}\right) / \sum_{j=1}^{93} K\left(\frac{j-j^{*}}{h}\right)$ in which $h$ is a bandwidth and $K$ is a kernel. In this regard, the so-called triweight kernel was utilized. The cross-validation criterion was also employed to evaluate the optimal value of $h$. The plots of $x_{i}(t)$ and their smoothed functions (as well as $\widetilde{y}_{i}=\left(y_{i} ; l_{y_{i}}\right)_{T}$ ) are given in Figs. 5-8. Consider the 
following univariate fuzzy functional regression model:

$$
\widetilde{y}_{i}=\widetilde{\alpha} \oplus \int_{1}^{93}\left(\widetilde{\beta}(t) \otimes x_{i}(t)\right) d t \oplus \widetilde{\epsilon}_{i}, i=2000, \ldots, 2019 .
$$

According to the proposed method, this gives the following fuzzy linear regression:

$$
\widetilde{y}_{i}=\widetilde{\alpha} \oplus\left(\frac{92}{N}\right) \bigoplus_{k=1}^{93}\left(\widetilde{\beta}\left(U_{k}\right) \otimes x_{i}\left(U_{k}\right)\right) \oplus \widetilde{\epsilon}_{i}, i=2000, \ldots, 2019,
$$

where $\widetilde{\beta}\left(U_{k}\right)$ generates $\boldsymbol{T} \boldsymbol{F} \boldsymbol{N} s, U_{1}, U_{2}, \ldots, U_{N}$ are independent random variables uniformly distributed over the interval $[1,93]$, and $N \in \mathbb{N}$ is a large number. Here, it was assumed that $N=100$. The results of performance evaluations are summarized in Table 3. In addition, Fig. 9 presents the $3 D$-plot of $\widetilde{\beta}(t)$. In order to evaluate the effect of functional predictors on the fuzzy response, the functional data was converted to scalar values (mean values over summer). Then, the proposed method was compared with some common fuzzy regression models (Hao and Chiang [35], Wang et al. [45]), Atalay et al. [3], Choi and Buckley [71], Choi and Yoon [72], Kula and Apaydin [73], Zeng et al. [74], D'Urso and Gastaldi [75] and Roldan Lopez de Hierro et al. [76])). For this purpose, let us consider the conventional fuzzy linear regression model in cases where $x_{i}(t) s$ are converted to mean values of $x_{i}$ :

$$
\widetilde{y}_{i}=\widetilde{\alpha} \oplus \int_{1}^{93}\left(\widetilde{\beta}(t) \otimes x_{i}\right) d t \oplus \widetilde{\epsilon}_{i}=\widetilde{\alpha} \oplus\left(\widetilde{\beta}^{\prime} \otimes x_{i}\right) \oplus \widetilde{\epsilon}_{i},
$$

where $\widetilde{\beta}^{\prime}=\int_{0}^{93}(\widetilde{\beta}(t)) d t$. The results of some common fuzzy univariate linear/nonlinear regression models are summarized in Table 3. A comparison among different methods indicates that the proposed method in this study led to more accurate results in terms of $M S M=0.70, M A R E=11.682, R M S E=9.22$ and $A U R O C C_{1} 5=$ 10.04. The accuracy of the proposed method along with other ones were also examined by comparing the corresponding $M_{\widetilde{y}}$ and $M_{\widetilde{y}}$ values, as shown in Figs. 10-11, further confirming the superiority of the hereby presented method over the other methods for this simulation example. Therefore, incorporation of functional data into a fuzzy regression model is expected to lead to more accurate performance measures compared to the conventional fuzzy regression models with scalar data.

\section{Conclusion}

Functional regression models are used to evaluate the complex relationship between repeatedly measured variables. In this paper, a regression model was built 
Table 3: Coefficients of the model and performance measures corresponding to some fuzzy regression techniques in Example 4.2.

\begin{tabular}{|c|c|c|c|c|c|}
\hline Method & Fuzzy coefficients & $M S M$ & $M A R E$ & $R M S E$ & $A U R O C C_{15}$ \\
\hline Choi and Yoon & $\widehat{\beta}_{0}=(14.77 ; 0.010)_{T}, \widehat{\beta}_{1}=(-30.11 ; 0.15)_{T}, \widehat{\beta}_{2}=(13.77 ; 0.12)_{T}$ & 0.51 & 17.05 & 15.46 & 15.94 \\
\hline Zeng et al. & $\widehat{\beta}_{0}=(15.78 ; 0.06)_{T}, \widehat{\beta}_{1}=(-31.78 ; 0.11)_{T}, \widehat{\beta}_{2}=(15.88 ; 0.01)_{T}$ & 0.54 & 15.83 & 13.73 & 14.17 \\
\hline Kula and Apaydin & $\widehat{\beta}_{0}=(15.88 ; 0.10)_{T}, \widehat{\beta}_{1}=(-33.55 ; 0.15)_{T}, \widehat{\beta}_{2}=(13.77 ; 0.12)_{T}$ & 0.54 & 16.82 & 13.73 & 15.42 \\
\hline Choi and Buckley & $\widehat{\beta}_{0}=(12.01 ; 0.06)_{T}, \widehat{\beta}_{1}=(-27.77 ; 0.24)_{T}, \widehat{\beta}_{2}=(118.80 ; 0.09)_{T}$ & 0.52 & 18.32 & 15.79 & 14.73 \\
\hline Wang et al. & $\hat{h}=0.372$ & 0.53 & 15.93 & 14.88 & 16.01 \\
\hline Hao and Chiang & $\begin{array}{c}\widehat{\widehat{\beta}}_{0}=(15.66 ; 0.03)_{T}, \widehat{\widehat{\beta}}_{1}=(-36.17 ; 0.08)_{T}, \widehat{\beta}_{2}=(16.49 ; 0.06)_{T}, \\
H=0.5, K=150, P=30\end{array}$ & 0.59 & 11.98 & 11.21 & 11.39 \\
\hline Proposed & $\widehat{\alpha}=(15.82 ; 0.12)_{T}, \widehat{\lambda}=2.35$ & 0.70 & 10.68 & 9.22 & 10.04 \\
\hline Roldan Lopez de Hierro et al. & $\widehat{\beta}_{0}=(15.92 ; 0.05)_{T}, \widehat{\beta}_{1}=(-32.29 ; 0.07)_{T}, \widehat{\beta}_{2}=(17.92 ; 0.06)_{T}$ & 0.57 & 13.82 & 10.61 & 11.96 \\
\hline D'Urso and Gastaldi & $\begin{array}{c}\widehat{y}(x)=\left(f(x) ; l_{f(x)}\right)_{T}, \\
f(x)=14.55-35.88 x+18.99 x^{2}, \\
l_{f(x)}=-5.407+8.602 x\end{array}$ & 0.51 & 17.05 & 15.46 & 15.94 \\
\hline Atalay et al. & $\widehat{h}=\widehat{k}_{1}=\widehat{k}_{2}=0.5$ & 0.56 & 15.42 & 14.27 & 13.88 \\
\hline
\end{tabular}
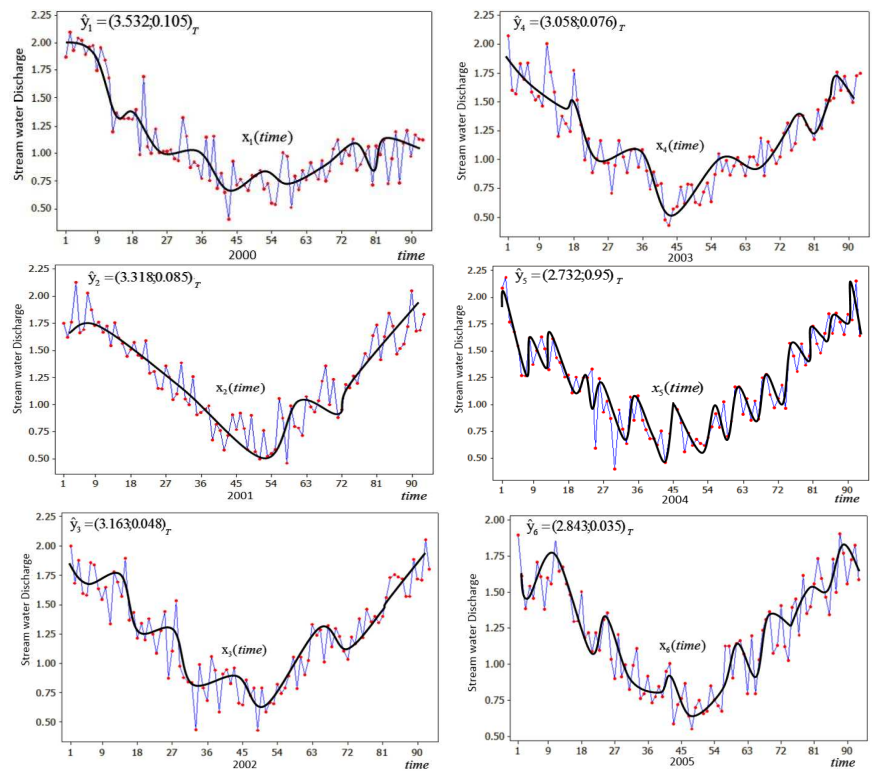

Figure 5: Time series plots of the stream water discharge and their smooth functions in Example $4.2(1)$.

for a functional fuzzy response where the predictors were functions. To this end, the concept of fuzzy integral of a fuzzy-valued function was first defined. Then, a fuzzy estimated value of the fuzzy integral of the fuzzy-valued function was proposed using the large numbers theorem. Then a regularization technique was adopted with absolute error deviation, SCAD penalty, and cross-validation criteria to evaluate the 

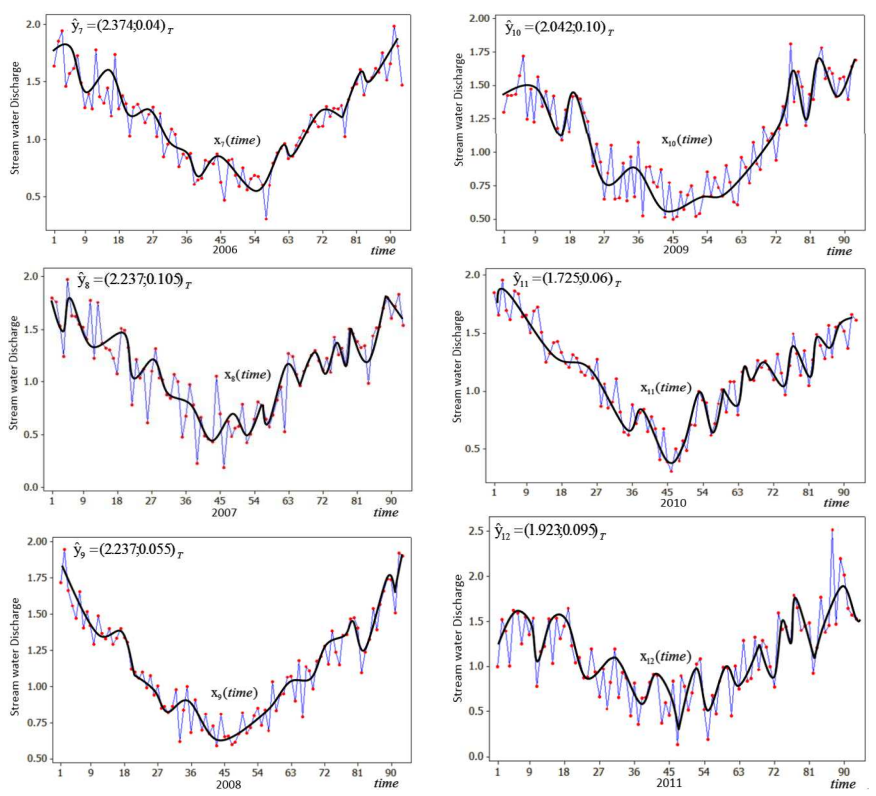

Figure 6: Time series plots of the stream water discharge and their smooth functions in Example $4.2(2)$.

coefficients and tuning constant of the fuzzy-valued function. The proposed regression model was subsequently examined according to several goodness-of-fit criteria via an applied example and a simulation study. The results were compared to those of some common fuzzy linear regression models in cases where the functional data was reduced to exact values. The findings clearly indicated the higher efficiency of the proposed method in this research over other techniques. The proposed method can be applied for virtually any kind of LR-fuzzy response. Further research works may focus on extending the proposed model to the the cases where the predictors are also fuzzy-valued functions. A sensitivity analysis with respect to outliers can represent another potential topic for further studies.

\section{Compliance with ethical standards}

Conflict of interest: The authors declare that they have no conflict of interest. Ethical standard: This article does not contain any studies with human participants or animals performed by the authors.

\section{CRediT authorship contribution statement:}

Gholamreza Hesamian: Conceptualization, Methodology, Writing-review \& edit- 

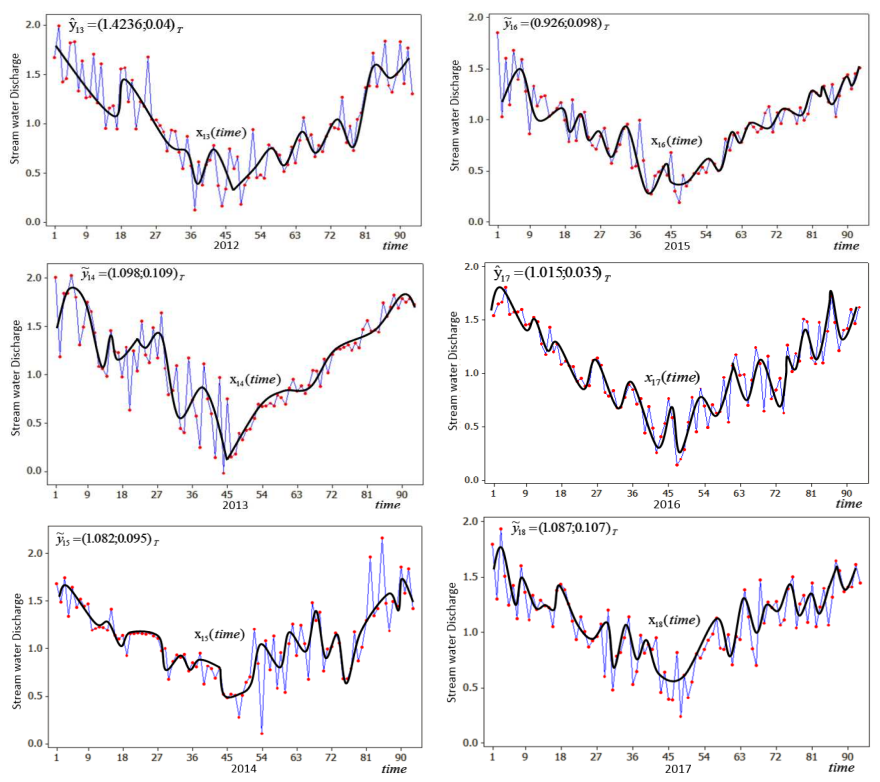

Figure 7: Time series plots of the stream water discharge and their smooth functions in Example $4.2(3)$.
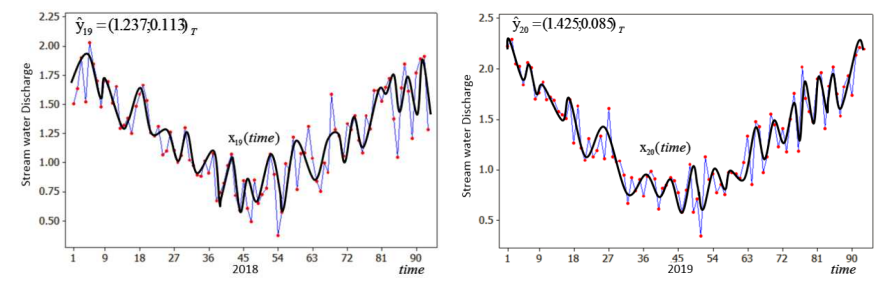

Figure 8: Time series plots of the stream water discharge and their smooth functions in Example $4.2(4)$.

ing. Mohammad Ghasem Akbari: Software, Validation, Investigation.

\section{References}

[1] Chukhrova, N. \& A. Johannssen (2019): Fuzzy regression analysis: Systemtic review and bibliography. Applied Soft Computing, 84. https://doi.org/10.1016/j.asoc.2019.105708

[2] Amiri, M.J., A.R. Zarei, J. Abedi-Koupai \& S. Eslamian (2019): The performance of fuzzy regression method for estimating of reference evapotranspiration 


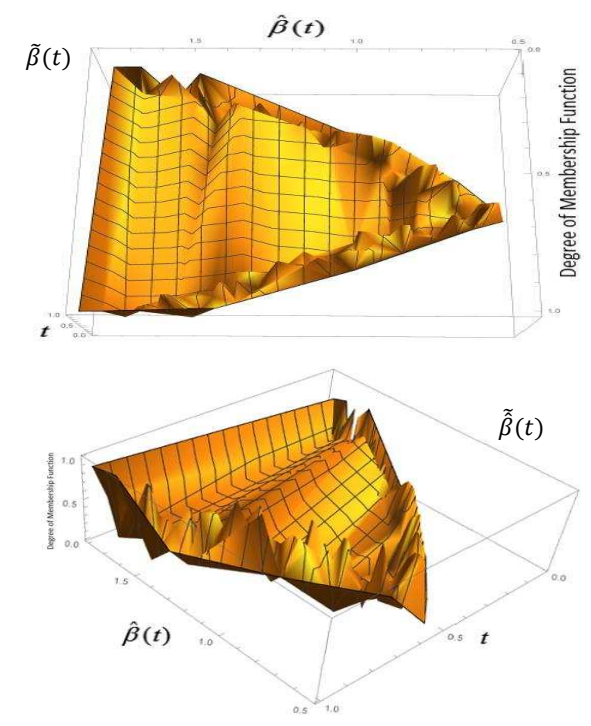

Figure 9: 3D-plot of $\widetilde{\beta}(t)$ and $\widetilde{\widehat{\beta}}(t)$ in Example 4.2 .

under controlled environment. International Journal of Hydrology Science and Technology, 9, pp. 28-38.

[3] Atalay, K.D., E. Eraslan \& M.O. Cinar (2015): A hybrid algorithm based on fuzzy linear regression analysis by quadratic programming for time estimation: An experimental study in manufacturing industry. Journal of Manufacturing Systems, 36, pp. 182-188.

[4] Chung, W. (2012): Using the fuzzy linear regression method to benchmark the energy efciency of commercial buildings. Applied Energy, 95, pp. 45-49.

[5] De Andres-Sanchez, J. (2016): Fuzzy regression analysis: An actuarial perspective. In: fuzzy statistical decision-making: Theory and applications, Studies in Fuzziness and Soft Computing, 343. Edited by C. Kahraman \& O. Kabak. Springer, Berlin, pp. 175-201.

[6] Hassanpour, H., H.R. Maleki \& M.A. Yaghoobi (2011): A goal programming approach to fuzzy linear regression with fuzzy input-output data. Soft Computing, 15 , pp. $1569-1580$.

[7] Hong, T. \& P. Wang (2014): Fuzzy interaction regression for short term Load forecasting. Fuzzy Optimization and Decision Making, 13(1), pp. 91-103. 

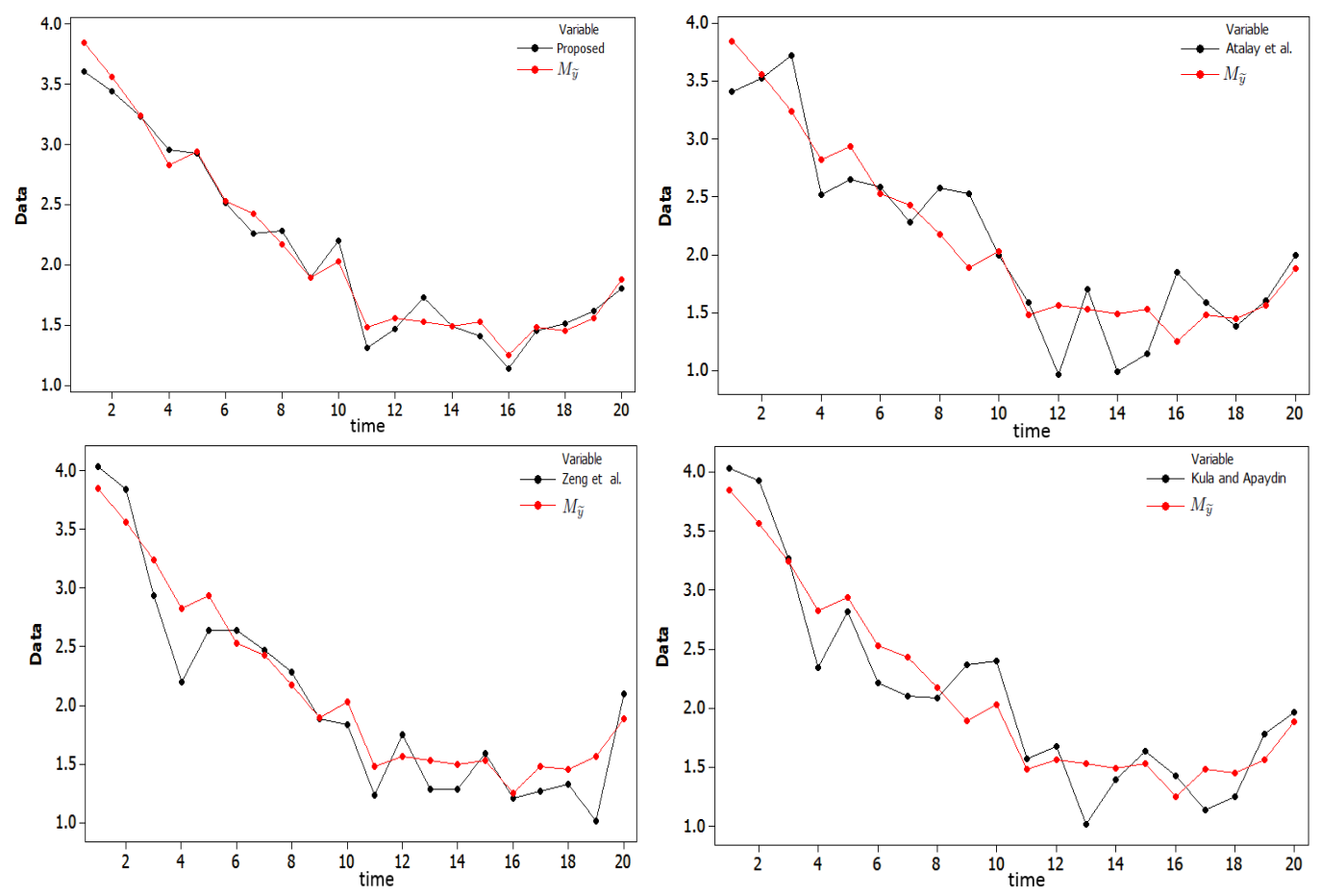

Figure 10: Comparison of $M_{\widetilde{\widehat{y}}}$ versus $M_{\widetilde{y}}$ in Example 4.2 (1). 

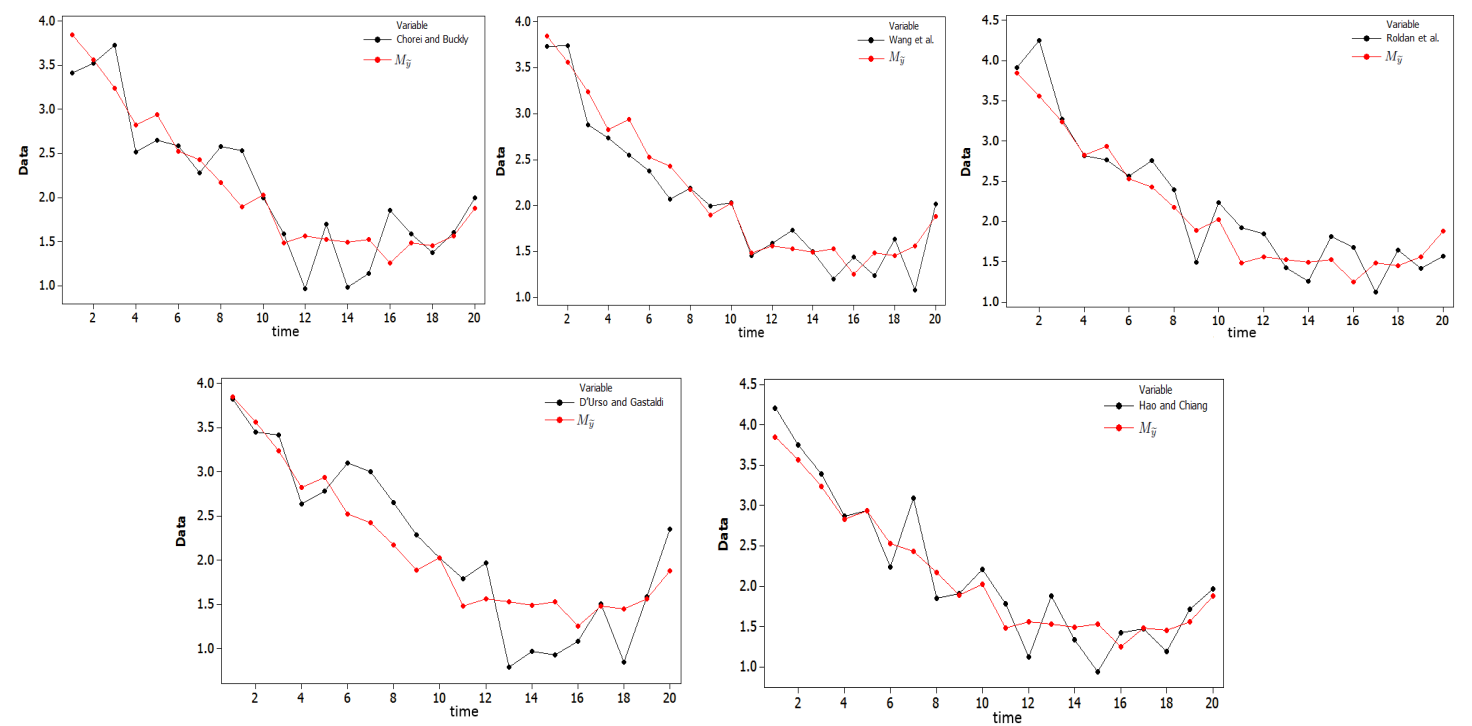

Figure 11: Comparison of $M_{\widetilde{y}}$ versus $M_{\widetilde{y}}$ in Example $4.2(2)$.

[8] Kocadagli, O. (2013): A novel non-linear programming approach for estimating CAPM beta of an asset using fuzzy regression. Expert Systems with Applications, 40, pp. 858-865.

[9] Modarres, M., E. Nasrabadi \& M.M. Nasrabadi (2005): Fuzzy linear regression models with least square errors. Applied Mathematics and Computation, 163, pp. $977-989$.

[10] Muzzioli, S., A. Ruggieri \& B. De Baets (2015): A comparison of fuzzy regression methods for the estimation of the implied volatility smile function. Fuzzy Sets and Systems, 266, pp. 131-143.

[11] Shakouri Ganjavi, H., R. Nadimi \& S.F. Ghaderi (2017): Investigation on objective function and assessment rule in fuzzy regressions based on equality possibility, fuzzy union and intersection concepts. Computers $\&$ Industrial Engineering, 110, pp. 207-215.

[12] Tanaka, H. \& H. Ishibuchi (1992): Possibilistic Regression analysis based on linear programming. In: fuzzy regression analysis. Edited by J. Kacprzyk \& M. Fedrizzi. Omnitech Press, Warsaw, and Physica-Verlag, Heidelberg, pp. 47-60. 
[13] Zolfaghari, Z.S., M. Mohebbi \& M. Najariyan (2014): Application of fuzzy linear regression method for sensory evaluation of fried donut. Applied Soft Computing, 22 , pp. $417-423$.

[14] Jiang, H., C.K. Kwong \& W.Y. Park (2017): Probabilistic fuzzy regression approach for preference modeling. Engineering Applications of Artifcial Intelligence, 64, pp. 286-294.

[15] Akbari, M.G. \& G. Hesamian (2019): Elastic net oriented to fuzzy semiparametric regression model with fuzzy explanatory variables and fuzzy Responses. IEEE Transactions on Fuzzy Systems, 17, pp. 2433-2442.

[16] Akbari, M.G. \& G. Hesamian (2019): A partial-robust-ridge-based regression model with fuzzy predictors-responses. Journal of Computational and Applied Mathematics, 351, pp. 290-301.

[17] Chan, K.Y. \& U. Engelke (2015): Fuzzy regression for perceptual image quality assessment. Engineering Applications of Artifcial Intelligence, 43, pp. 102-110.

[18] Celmins, A. (1987): Least squares model fitting to fuzzy vector data. Fuzzy Sets and Systems, 22, pp. 245-269.

[19] D'Urso, P. \& R. Massari (2013): Weighted least squares and least median squares estimation for the fuzzy linear regression analysis. Metron, 71, pp. 279306.

[20] Hesamian, G., M.G. Akbari \& M. Asadollahi (2017): Fuzzy semi-parametric partially linear model with fuzzy inputs and fuzzy outputs. Expert Systems with Applications, 71, pp. 230-239.

[21] Khan, U.T. \& C. Valeo (2015): A new fuzzy linear regression approach for dissolved oxygen prediction. Hydrological Sciences Journal, 60, pp. 1096-1119.

[22] Kim, I.K., W.J. Lee, J. H. Yoon \& S. H. Choi (2016): Fuzzy regression model using trapezoidal fuzzy numbers for re-auction data. International Journal of Fuzzy Logic and Intelligent Systems, 16, pp. 72-80.

[23] Liu, Y., Y. Chen, J. Zhou \& S. Zhong (2015): Fuzzy linear regression models for QFD using optimized h Values. Engineering Applications of Artifcial Intelligence, 39, pp. 45-54. 
[24] Roldan Lopez de Hierro, A.F., J. Martinez-Moreno, C. Aguilar-Pena \& C. Roldan Lopez de Hierro (2016b): Estimation of a fuzzy regression model using fuzzy distances. IEEE Transactions on Fuzzy Systems, 24, pp. 344-359.

[25] Yoon, J.H., S.H. Choi \& P. Grzegorzewski (2016): On asymptotic properties of the multiple fuzzy least squares estimator. In: Soft Methods for Data Science, Advances in Intelligent Systems and Computing, 456. Edited by M. B. Ferraro, P. Giordani, B. Vantaggi, M. Gagolewski, M. A. Gil, P. Grzegorzewski \& O. Hryniewicz. Springer, Berlin, pp. 525-532.

[26] Zhou, J., H. Zhang, Y. Gu \& A.A. Pantelous (2018): Affordable levels of house prices using fuzzy linear regression analysis: the case of shanghai. Soft Computing, 22, pp. 5407-5418.

[27] Zuo, H., G. Zhang, W. Pedrycz, V. Behbood \& J. Lu (2018): Granular fuzzy regression domain adaptation in Takagi-Sugeno fuzzy models. IEEE Transactions on Fuzzy Systems, 26, pp. 847-858.

[28] Icen, D. \& H. Demirhan (2016): Error measures for fuzzy linear regression: Monte carlo simulation approach. Applied Soft Computing, 46, pp. 104-114.

[29] Chachi, J. (2019): A weighted least-squares fuzzy regression for crisp input-fuzzy output data. IEEE Transactions on Fuzzy Systems, 17, pp. 739-748.

[30] Buckley, J. J. \& Y. Hayashi (1994): Fuzzy genetic algorithm and applications. Fuzzy Sets and Systems, 61, pp. 129-136.

[31] Chan, K.Y. \& C.K. Kwong (2013): Modeling of epoxy dispensing process using a hybrid fuzzy regression approach. The International Journal of Advanced Manufacturing Technology, 65, pp. 589-600.

[32] Chan, K.Y., H.K. Lam, C.K.F. Yiu \& T. S. Dillon (2017): A flexible fuzzy regression method for addressing non-linear uncertainty on aesthetic quality assessments. IEEE Transactions on Systems, Man, and Cybernetics: Systems, 47, pp. 2363-2377.

[33] Gholizadeh, H., N. Javadian \& H. Fazlollahtabar (2018): Fuzzy regression integrated with genetic-tabu algorithm for prediction and optimization of a turning process. The International Journal of Advanced Manufacturing Technology, 96, pp. 2781-2790. 
[34] Hu, Y.C. (2014): Multilayer perceptron for robust non-linear interval regression analysis using genetic algorithms. The Scientific World Journal, Article ID 970931, pp. 1-8.

[35] Hao, P.Y. \& J.H. Chiang (2008): Fuzzy regression analysis by support vector learning approach. IEEE Transactions on Fuzzy Systems, 16, pp. 428-441.

[36] Huang, C.H. (2012): A reduced support vector machine approach for interval regression analysis. Information Sciences, 217, pp. 56-64.

[37] Megri, F., A.C. Megri \& R. Djabri (2016): An integrated fuzzy support vector regression and the particle swarm optimization algorithm to predict indoor thermal comfort. Indoor and Built Environment, 25, pp. 1248-1258.

[38] Wieszczy, P. \& P. Grzegorzewski (2016): Support vector machines in fuzzy regression. In: challenging problems and solutions in intelligent systems, Studies in Computational Intelligence, 634. Edited by G. de Tre, P. Grzegorzewski, J. Kacprzyk, J. W. Owsinski, W. Penczek \& S. Zadrozny. Springer, Switzerland, pp. 103-138.

[39] Azadeh, A., S. M.H.M. Alajdad \& T.A. Bioki (2014): A neuro-fuzzy regression approach for estimation and optimization of gasoline consumption. International Journal of Services and Operations Management, 17, pp. 221-256.

[40] He, Y.L., C.H. Wei, H. Long, R.A.R. Ashfaq \& J.Z. Huang (2017): Random weight network-based fuzzy non-linear regression for trapezoidal fuzzy number data. Applied Soft Computing, 70, pp. 959-979.

[41] Liu, H.T., J. Wang, Y.L. He \& R.A.R. Ashfaq (2017): Extreme learning machine with fuzzy input and fuzzy output for fuzzy regression. Neural Computing and Applications, 28, pp. 3465-3476.

[42] Pehlivan, N.Y. \& A. Apaydin (2016): Fuzzy radial basis function network for fuzzy regression with fuzzy input and fuzzy output. Complex $\&$ Intelligent Systems, 2, pp. 61-73.

[43] Roh, S.K., T.C. Ahn \& W. Pedrycz (2012): Fuzzy linear regression based on polynomial neural networks. Expert Systems with Applications, 39, pp. 89098928. 
[44] Cheng, C.B. \& E.S. Lee (1999). Non-parametric fuzzy regression K-NN and kernel smoothing techniques. Computers and Mathematics with Applications, 38 , pp. 239-251.

[45] Wang, N., W.X. Zhang \& C.L . Mei (2007): Fuzzy non-parametric regression based on local linear smoothing technique. Information Sciences, 177, pp. 3882 3900 .

[46] Hesamian, G. and Akbar, M.G. (2020). A Robust Varying Coefficient Approach to Fuzzy Multiple Regression Model. Journal of Computational and Applied Mathematics, 375, pp 1-13.

[47] Yang, Z. and Yin, Y. (2013). Robust Fuzzy Varying Coefficient Regression Analysis with Crisp Inputs and Gaussian Fuzzy Output. Journal of Computing Science and Engineering, 7, pp. 263-271.

[48] James G. (2002): Generalized linear models with functional predictors. Journal of Royal Statistical Association: Series B., 64, pp. 411-432.

[49] Ferraty F. \& P. Vieu. Nonparametric Functional Data Analysis: Theory and Practice. Springer Science \& Business Media, 2006.

[50] Goldsmith J. \& F. Scheipl (2014): Estimator selection and combination in scalar-on-function regression. Computational Statistics and Data Analysis, 70, pp. 362372 .

[51] Ramsay J.O. \& B. W. Silverman. Functional Data Analysis, Wiley, New York, 2006.

[52] Faraway J. (1997): Regression Analysis for a Functional Response. Technometrics, 39, pp. 254261.

[53] Chiou J.M., H.G. Mller \& J.L. Wang (2004): Functional Response Models. Statistica Sinica, 14, pp. 675693.

[54] Ramsay, J.O. \& C.J. Dalzell (1991): Some Tools for Functional Data Analysis. Journal of the Royal Statistical Society. Series B., 53, pp. 539-572.

[55] Hastie, T. \& C. Mallows (1993): A statistical view of some chemometrics regression tools. Technometrics, 35, pp. 140-143.

[56] Hall P. \& J. L. Horowitz (2007): Methodology and convergence rates for functional linear regression. Annals of Statistics, 35, pp. 7091. 
[57] Yuan M. \& T. Cai (2010): A reproducing kernel hilbert space approach to functional linear regression. Annals of Statistics, 38, pp. 3412-3444.

[58] Febrero-Bande M. \& M.L. Oviedo de la Fuente (2012): Statistical computing in functional data analysis: The $R$ package. Journal of Statistical Software, 51, pp. $1-28$

[59] McLean M.W., G. Hooker, A. M. Staicu, F. Scheipl, \& D. Ruppert (2014): Functional generalized additive models. Journal of Computational and Graphical Statistics, 23, pp. 249-269.

[60] Usset, J., A. M. Staicu \& Maity, A. (2016): Interaction models for functional regression. Computational Statistics and Data Analysis, 94, pp. 317330.

[61] Reiss P.T., J. Goldsmith, H. L. Shang \& R. T. Ogden (2017): Methods for scalar-on-function regression. International Statistical Review, 85, pp. 228-249.

[62] Chiou J.M,. Yang, Y.F., Chen, Y.T. (2016): Multivariate functional linear regression and prediction. Journal of Multivariate Analysis, 146, pp. 301-312.

[63] Fan Y. Y., N. Foutz, G. M. James \& W. Jank (2014). Functional response additive model estimation with online virtual stock markets. Annals of Applied Statistics, 8, pp. 2435-2460.

[64] Radchenko P., X. Qiao \& G.M. James (2015) Index models for sparsely sampled functional data. Journal of American Statistical Association, 110, pp. 824836.

[65] Hesamian, G. \& M. Shams (2016): Parametric testing statistical hypotheses for fuzzy random variables. Soft Computing, 20, pp. 1537-1548.

[66] Lee, K.H. First Course on Fuzzy Theory and Applications, Springer-Verlag, Berlin, 2005.

[67] Simonnet M. The Strong Law of Large Numbers. In: Measures and Probabilities. Universitext. Springer, New York, NY, 1996.

[68] Wahba, G. Spline Models for Observational Data; society for industrial and applied mathematics. Siam, 1990.

[69] Trott, M. The Mathematica Guidebook For Programming, Springer-Verlag, New York, 2004. 
[70] Sugeno, M. (1985): An Introductory Survey of Fuzzy Control. Information Science, 36 , pp. 59-83.

[71] Choi, S.H. \& J.J. Buckley (2008): Fuzzy Regression Using Least Absolute Deviation Estimators. Soft Computing, 12, pp. 257-263.

[72] Choi, S.H. \& J.H. Yoon (2010): General fuzzy regression using least squares method. International Journal of Systems Science, 41, pp. 477-485.

[73] Kula, K. \& A. Apaydin (2008): Fuzzy robust regression analysis based on the ranking of fuzzy sets. International Journal of Uncertainty, Fuzziness and Knowledge-Based Systems, 16, pp. 663-681.

[74] Zeng, W., Q. Feng \& J. Li (2017): Fuzzy least absolute linear regression. Applied Soft Computing, 52, pp. 1009-1019.

[75] D'Urso, P. \& T. Gastaldi (2002). An order-wise polynomial regression procedure for fuzzy data. Fuzzy Sets and Systems, 130, pp. 1-19.

[76] Roldan Lopez de Hierro, A.F., J. Martinez-Moreno, C. Aguilar-Pena \& C. Roldan Lopez de Hierro (2016a): A Fuzzy Regression Approach using Bernstein Polynomials for the Spreads: Computational Aspects and Applications to Economic Models. Mathematics and Computers in Simulation, 128, pp. 13-25.

[77] Ryan, B.F., Joiner, B.L. and Cryer, J.D. MINITAB Handbook: Update for Release 16. Brooks/Cole Publishing Co., Pacific Grove, CA, USA, 2012. 
Figures
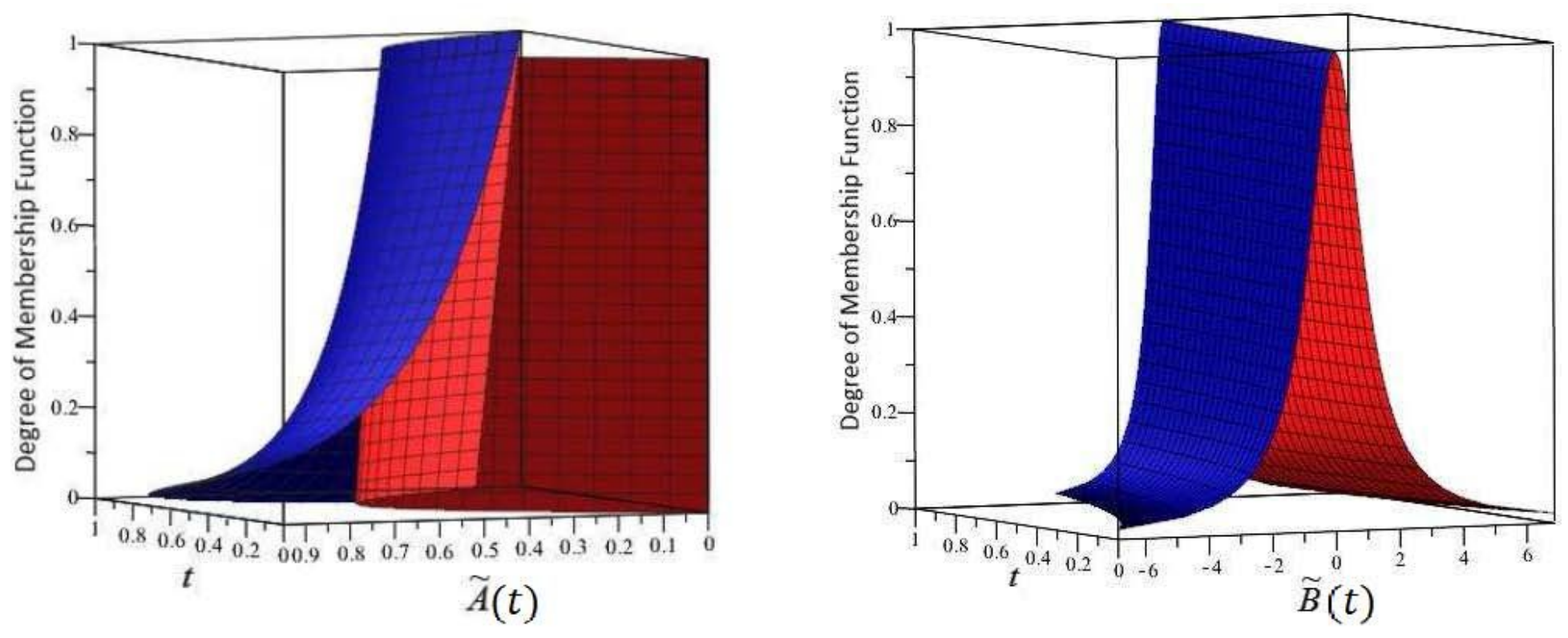

Figure 1

"Please see the Manuscript PDF file for the complete figure caption". 

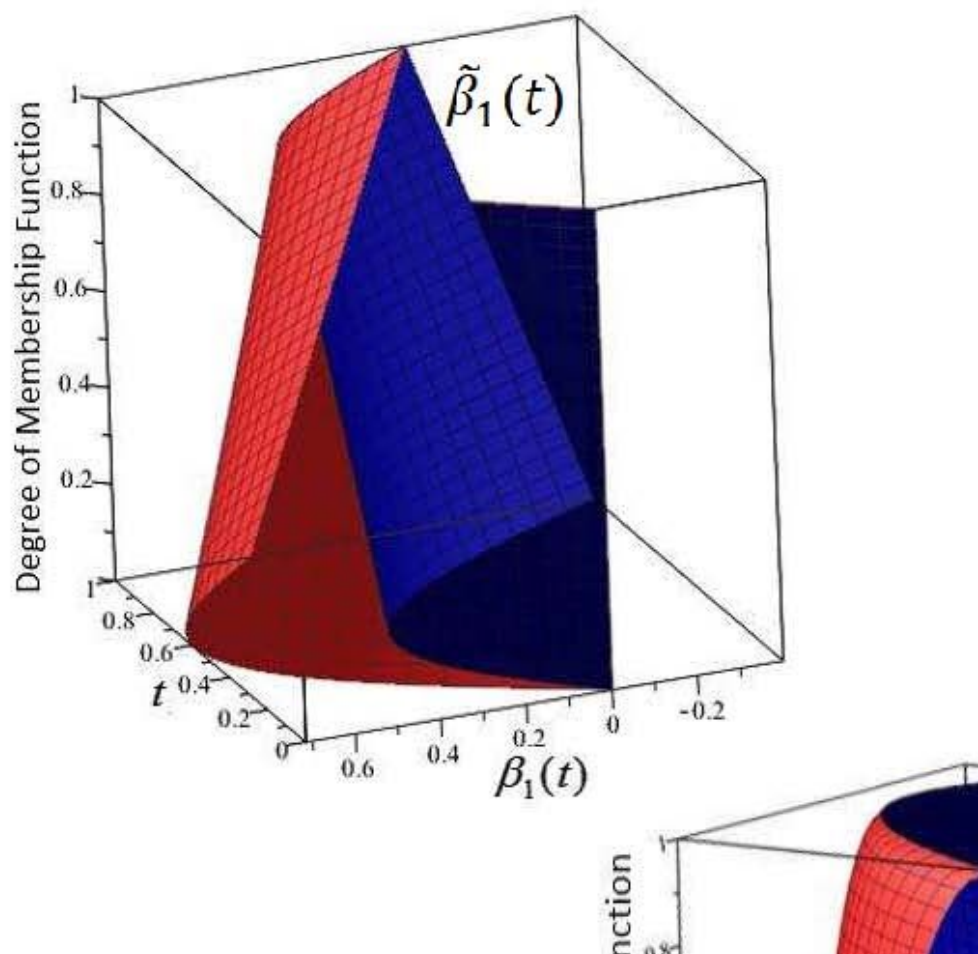

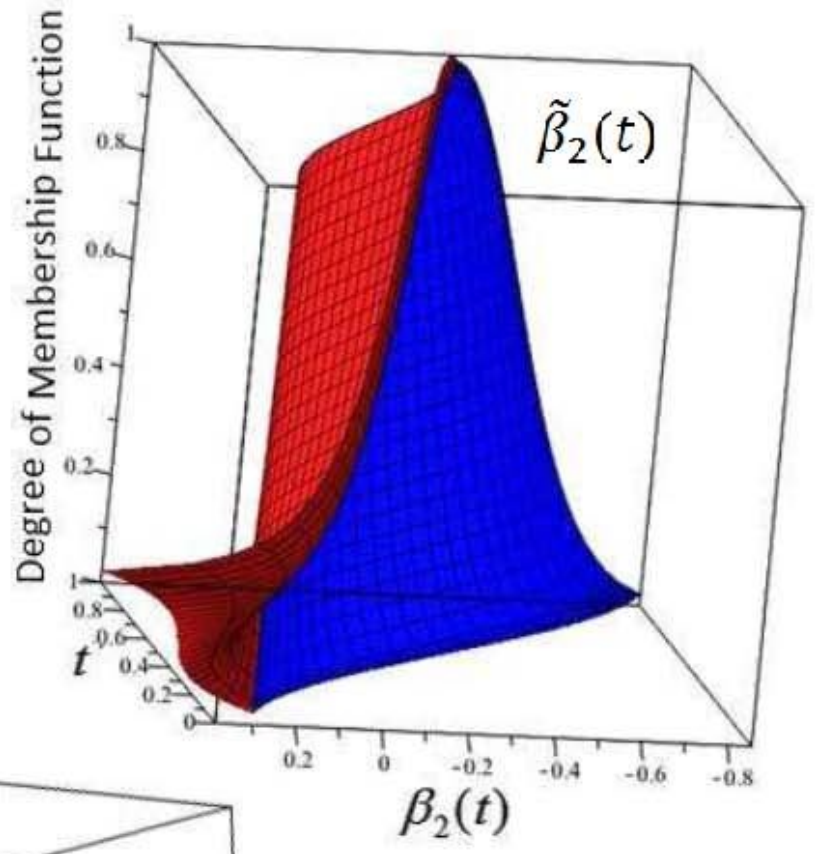

Figure 2

"Please see the Manuscript PDF file for the complete figure caption". 

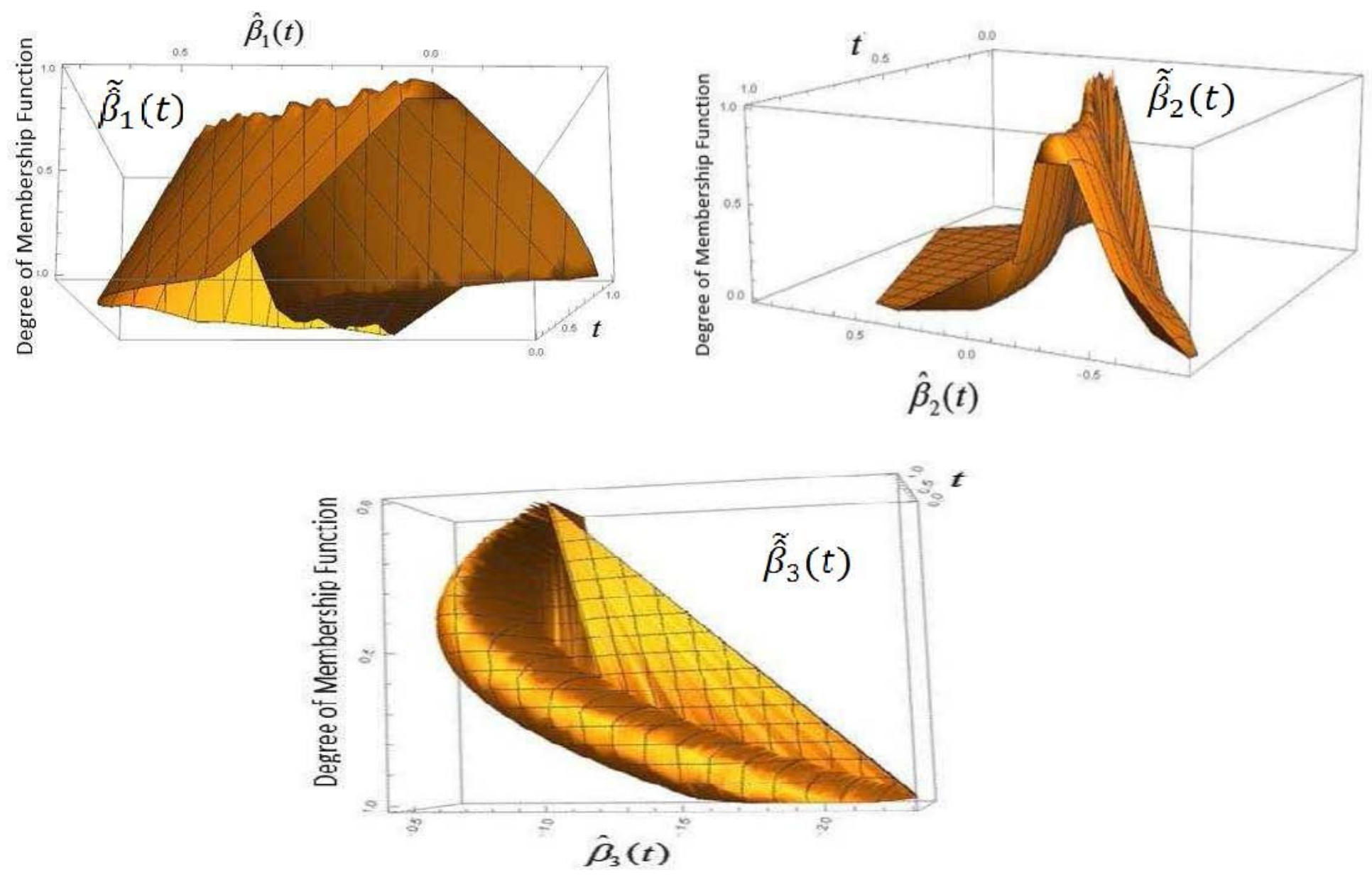

Figure 3

"Please see the Manuscript PDF file for the complete figure caption". 


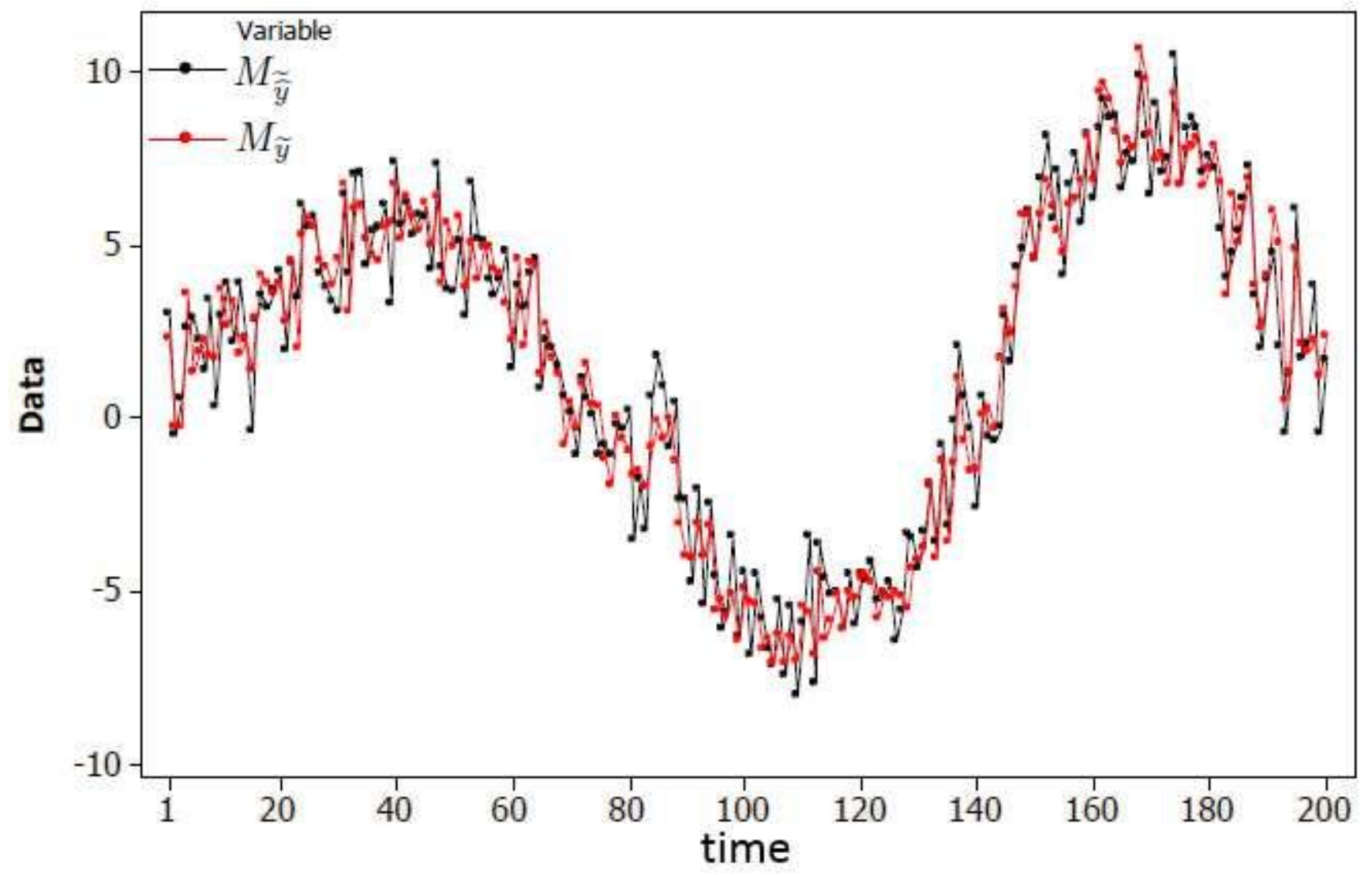

Figure 4

"Please see the Manuscript PDF file for the complete figure caption". 

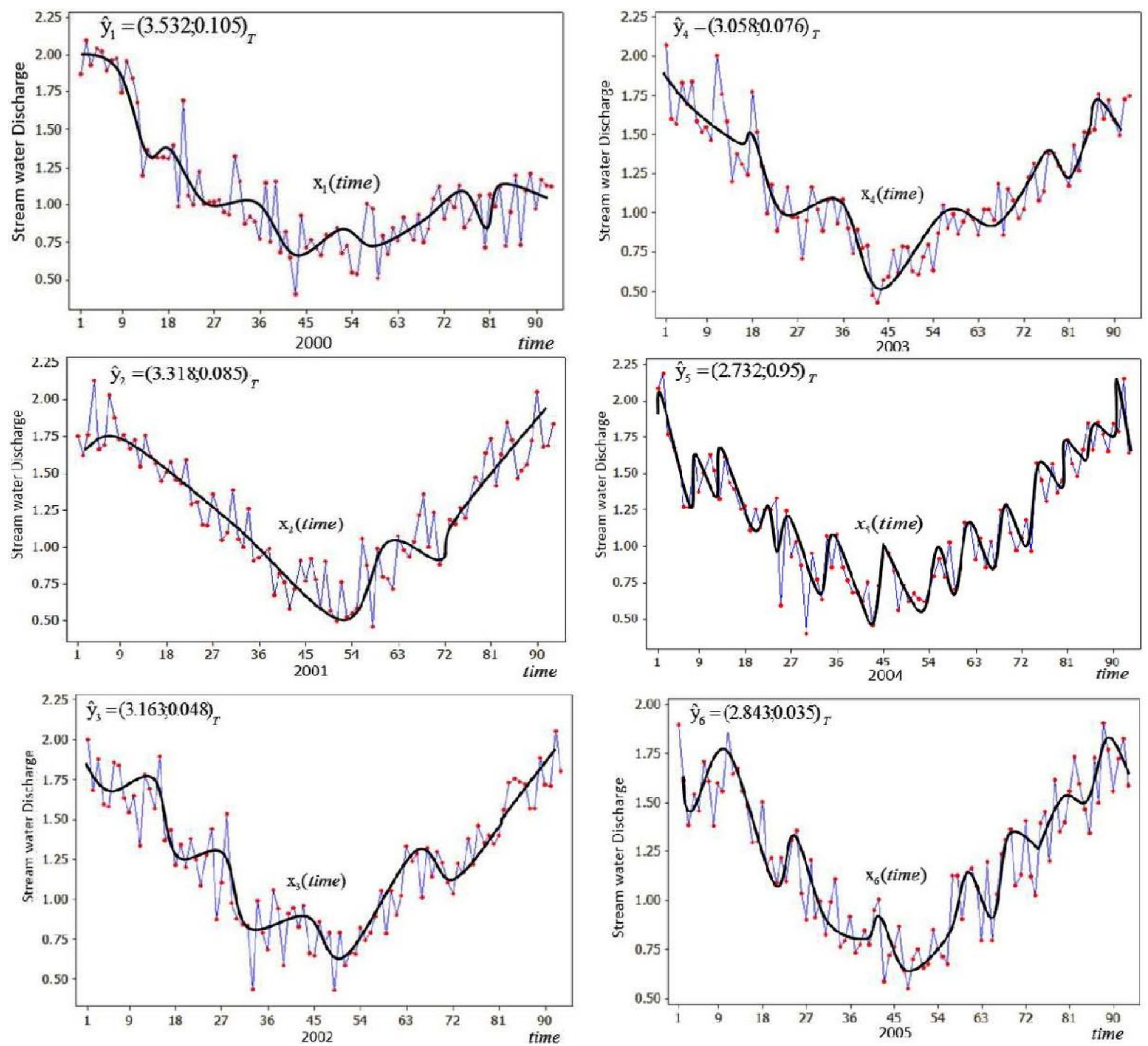

Figure 5

Time series plots of the stream water discharge and their smooth functions in Example 4.2(1). 



Figure 6

Time series plots of the stream water discharge and their smooth functions in Example 4.2(2). 

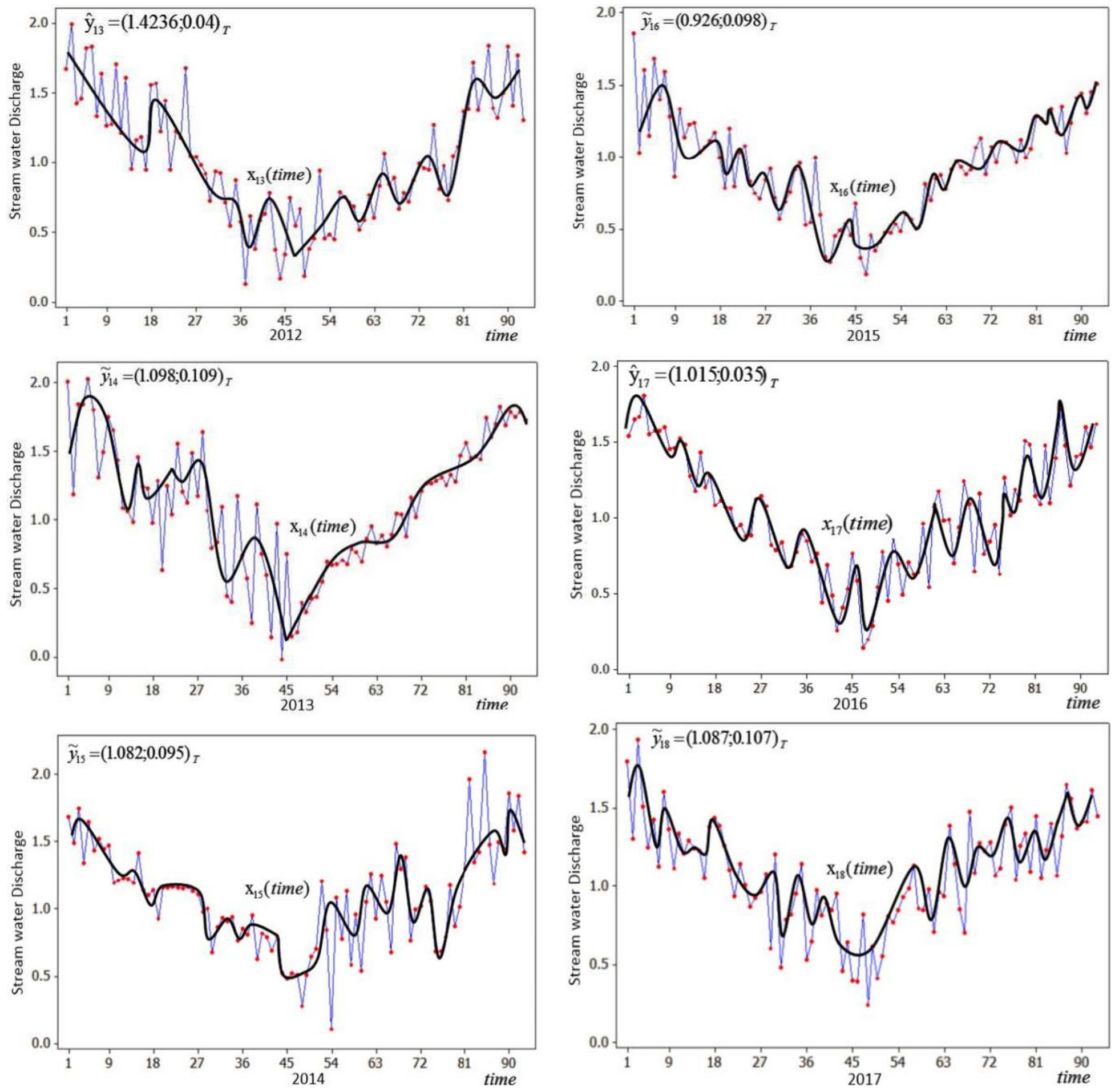

Figure 7

Time series plots of the stream water discharge and their smooth functions in Example 4.2(3). 

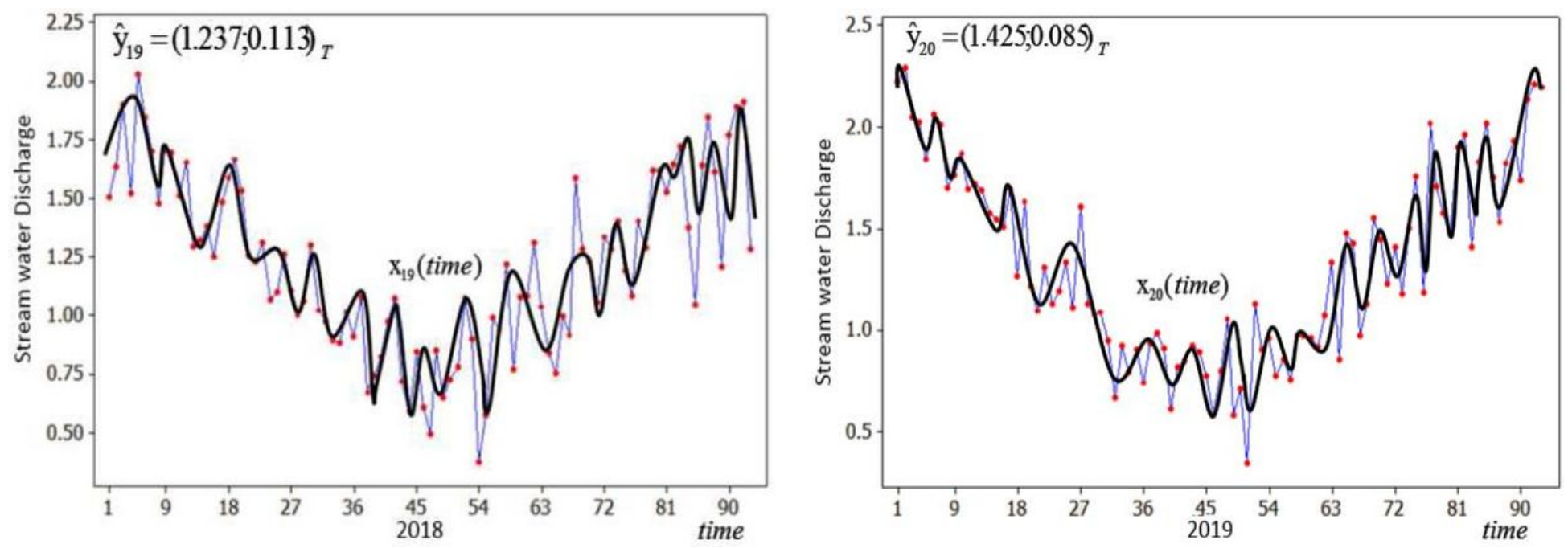

Figure 8

Time series plots of the stream water discharge and their smooth functions in Example 4.2(4). 

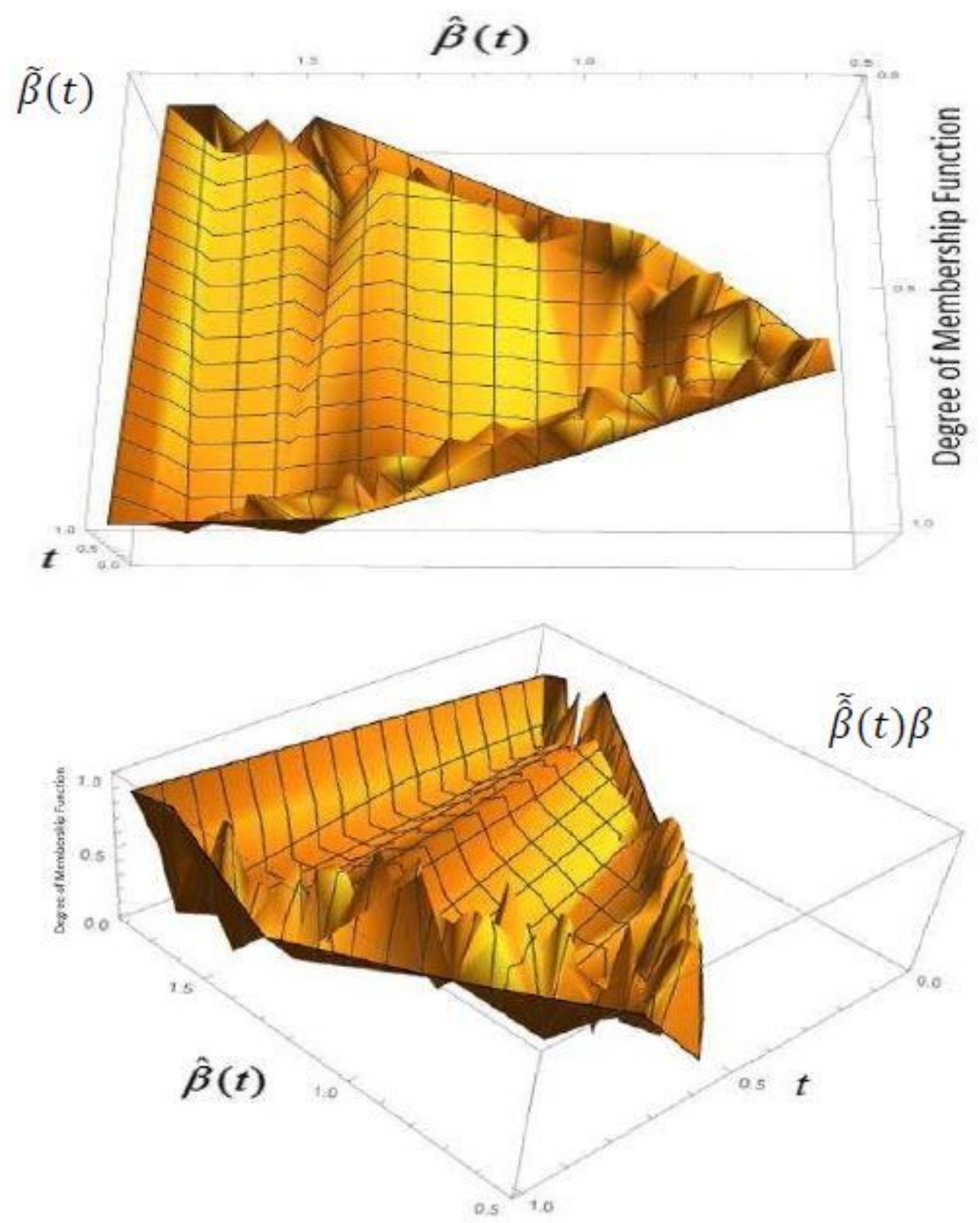

Figure 9

"Please see the Manuscript PDF file for the complete figure caption". 

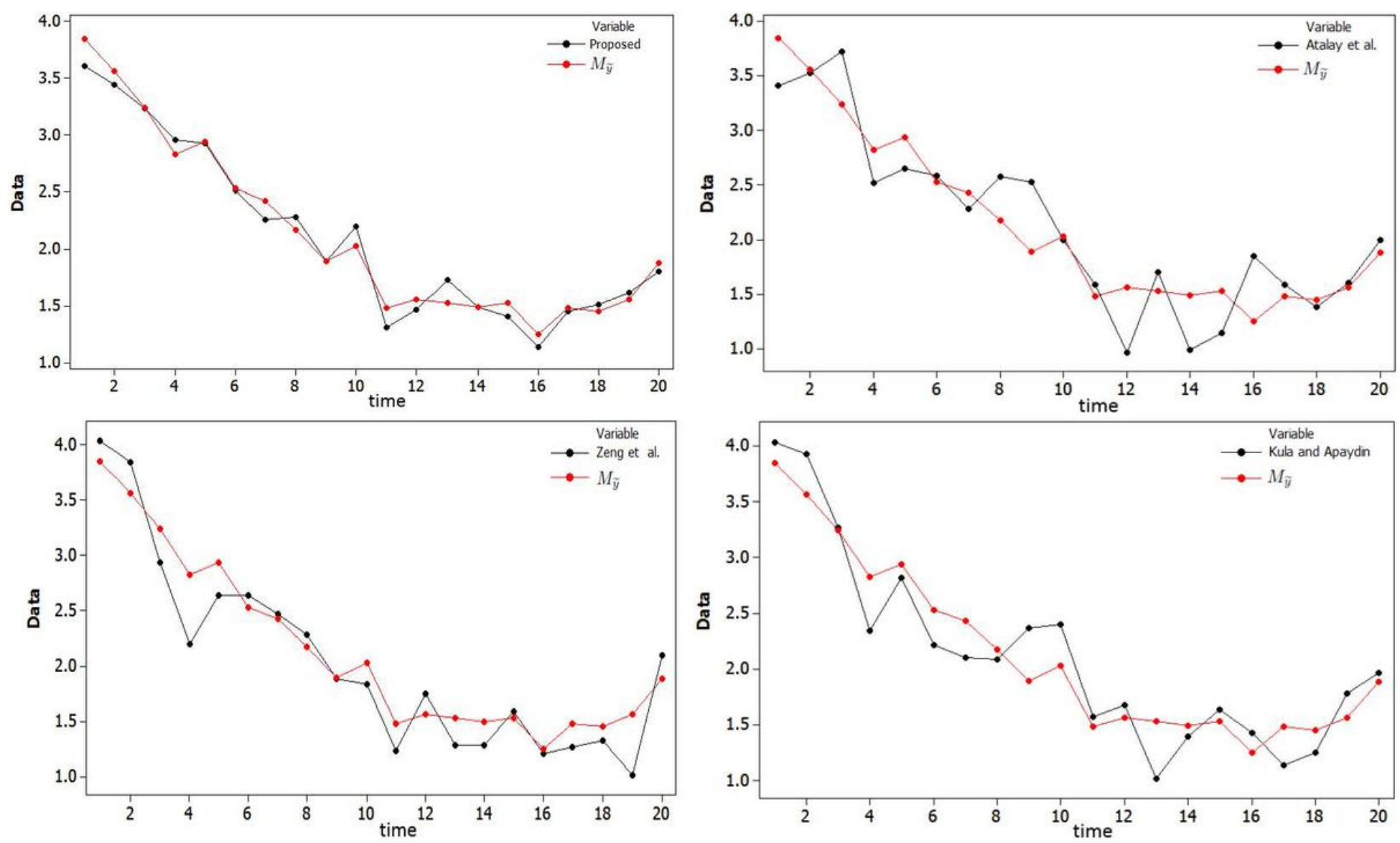

Figure 10

"Please see the Manuscript PDF file for the complete figure caption". 

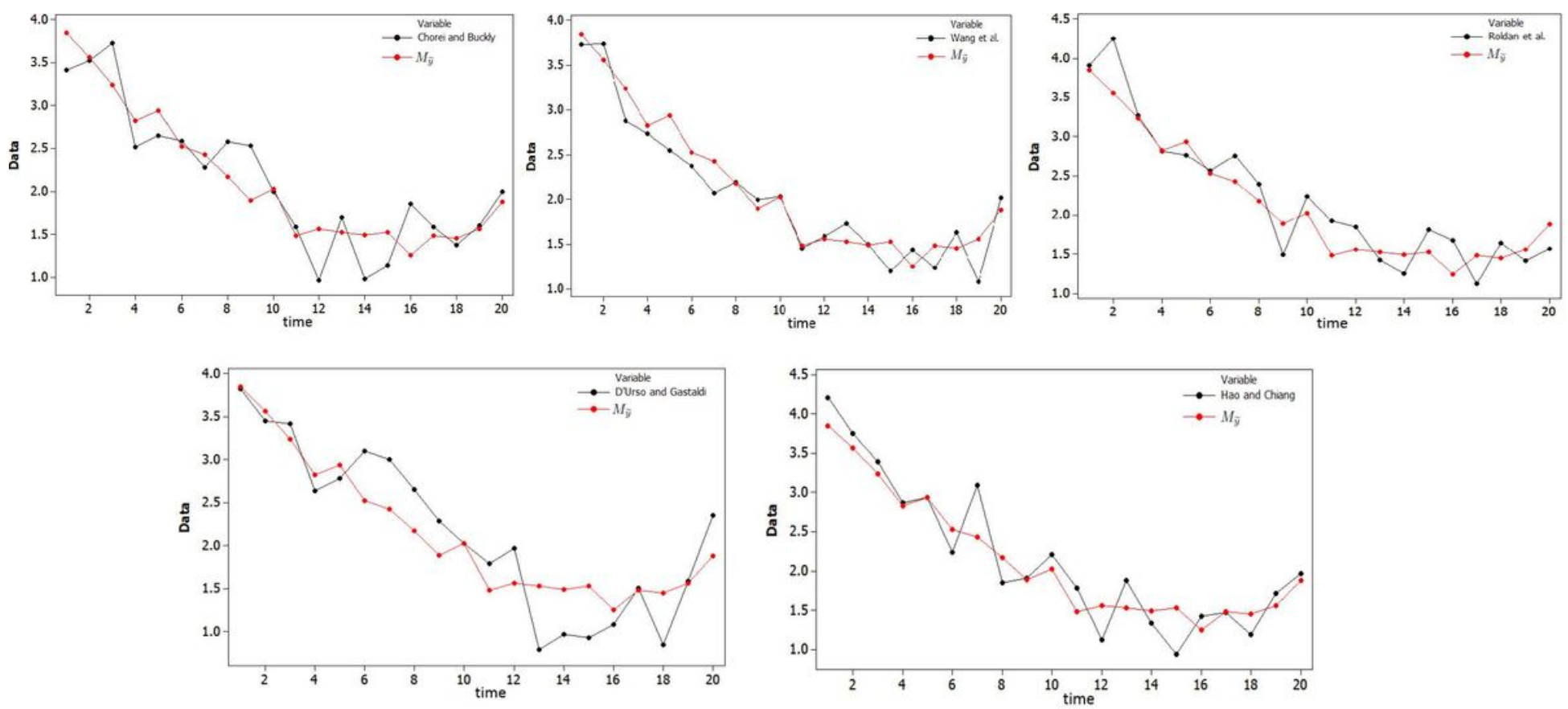

Figure 11

"Please see the Manuscript PDF file for the complete figure caption". 